\title{
Heavy element abundances in cool dwarf stars: An implication for the evolution of the Galaxy*
}

\author{
L. Mashonkina ${ }^{1,2,3}$ and T. Gehren ${ }^{2}$ \\ 1 Department of Astronomy, Kazan State University, Kremlevskaya 18, Kazan 8, 420008, Russia \\ 2 Institut für Astronomie und Astrophysik der Universität München, Scheinerstr. 1, 81679 München, Germany \\ 3 Max-Planck-Institut für Astrophysik, Karl-Schwarzschild-Straße 1, 85740 Garching, Germany
}

Received 16 March 2001 / Accepted 4 July 2001

\begin{abstract}
We present revised strontium, barium and europium abundances for 63 cool stars with metallicities $[\mathrm{Fe} / \mathrm{H}]$ ranging from -2.20 to 0.25 . The stellar sample has been extracted from Fuhrmann's lists (1998, 2001). It is confined to main-sequence and turnoff stars. The results are based on NLTE line formation obtained in differential model atmosphere analyses of spectra that have a typical S/N of 200 and a resolution of 40000 to 60000 . The element abundance ratios reveal a distinct chemical history of the halo and thick disk compared with that of the thin disk. Europium is overabundant relative to iron and barium in halo and thick disk stars suggesting that during the formation of these galactic populations high-mass stars exploding as SNe II dominated nucleosynthesis on a short time scale of the order of 1 Gyr. We note the importance of $[\mathrm{Eu} / \mathrm{Mg}]$ determinations for halo stars. Our analysis leads to the preliminary conclusion that $\mathrm{Eu} / \mathrm{Mg}$ ratios found in halo stars do not support current theoretical models of the $r$-process based on low-mass SNe; instead they seem to point at a halo formation time much shorter than $1 \mathrm{Gyr}$. A steep decline of $[\mathrm{Eu} / \mathrm{Fe}]$ and a slight decline of $[\mathrm{Eu} / \mathrm{Ba}]$ with increasing metallicity have been first obtained for thick disk stars. This indicates the start of nucleosynthesis in the lower mass stars, in SN I and AGB stars, which enriched the interstellar gas with iron and the most abundant $s$-process elements. From a decrease of the Eu/Ba ratio by $\sim 0.10 \ldots 0.15$ dex the time interval corresponding to the thick disk formation phase can be estimated. The step-like change of element abundance ratios at the thick to thin disk transition found in our previous analysis (Mashonkina \& Gehren 2000) is confirmed in this study: [Eu/Ba] and $[\mathrm{Eu} / \mathrm{Fe}]$ are reduced by $\sim 0.25$ dex and $\sim 0.15$ dex, respectively; $[\mathrm{Ba} / \mathrm{Fe}]$ increases by $\sim 0.1$ dex. This is indicative of an intermediate phase before the early stage of the thin disk developed, during which only evolved middle and low mass $\left(<8 M_{\odot}\right)$ stars contributed to nucleosynthesis. Our data provide an independent method to calculate the duration of this phase. The main $s$-process becomes dominant in the production of heavy elements beyond the iron group during the thin disk evolution. We find that in the thin disk stars Ba/Fe ratios increase with time from $[\mathrm{Ba} / \mathrm{Fe}]=-0.06$ in stars older than $8 \mathrm{Gyr}$ to $[\mathrm{Ba} / \mathrm{Fe}]=0.06$ in stars that are between 2 and 4 Gyr old.
\end{abstract}

Key words. line: formation - nuclear reactions, nucleosynthesis, abundances - stars: abundances - stars: late-type - Galaxy: evolution

\section{Introduction}

Observations of heavy elements beyond the iron group in cool dwarf stars give useful information about nucleosynthesis in the Galaxy and also for some important parameters of the evolution of the Galaxy: initial mass function (IMF), star formation rate (SFR), and a timescale for the formation of Galactic stellar populations. Abundances

\footnotetext{
Send offprint requests to: L. Mashonkina, e-mail: Lyudmila.Mashonkina@ksu.ru

* Based on observations collected at the German Spanish Astronomical Center, Calar Alto, Spain.
}

of these elements in the solar system have contributions in differing proportions from two processes, the $s$ - and $r$-process of neutron capture. In turn, the overall $s$-process abundance pattern is best fitted by a combination of two $s$-process components: the main s-process which occurs during the thermally pulsing asymptotic giant branch (AGB) phase of low-mass stars $\left(2-4 M_{\odot}\right)$, and which dominates the $s$-process contribution to $\mathrm{Rb}$ and heavier elements; and the weak s-process which is thought to run in the cores of massive stars, $M \geq 10 M_{\odot}$, and which corresponds to the lighter elements with $A \leq 85$ (Käppeler et al. 1989). The $r$-process is associated with explosive 
conditions in SNe II. Progenitors of SN II have much shorter lifetimes compared with those of AGB stars, and there must be a delay in the onset of the main $s$-process nucleosynthesis compared with the production of $r$-nuclei. The europium to barium abundance ratio is particularly sensitive to whether nucleosynthesis of the heavy elements occured in the $s$ - or $r$-process. For the solar system matter $\log \varepsilon_{\mathrm{Eu}, \odot}-\log \varepsilon_{\mathrm{Ba}, \odot}=-1.67$ (Grevesse et al. 1996). The contributions of the $s$ - and $r$-process to the solar Ba abundance consist of $81 \%$ and $19 \%$ according to the most recent data of Arlandini et al. (1999), whereas $94 \%$ of the solar europium originated from the $r$-process. Thus, the solar abundance ratio of $\mathrm{Eu}$ to $\mathrm{Ba}$ contributed by the $r$-process relative to the total abundances, $[\mathrm{Eu} / \mathrm{Ba}]_{r}$ equals 0.70 . The oldest stars of the Galaxy are expected to carry a significant Eu overabundance of about 0.7 dex relative to barium, provided that the $r$-process in early Galaxy was similar to solar one. A clear break in the run of [Eu/Ba] ratios with overall metallicity should signal the onset of the contribution to barium by AGB stars, and the location of this break provides an independent estimate for the timescale of star formation during the early stages of Galactic evolution.

In our previous analysis (Mashonkina \& Gehren 2000, thereafter Paper I) we have already presented Ba and Eu abundances in cool dwarf stars. An important advantage of that study was provided by taking into account the membership of individual stars in particular stellar populations of the Galaxy. The existence of the dynamically hot and metal-poor halo stellar population and the dynamically cool and metal-rich disk population was outlined by Eggen et al. (1962) on the base of dynamical and chemical data. This conclusion was supported by many of the later observational results. Gilmore \& Reid (1983) offered the first evidence for the existence of another Galactic stellar population, the thick disk. The properties of the thick disk place it between those of the halo and the thin disk, and the key question is whether it is related to either of them in terms of the Galaxy's chemical and dynamical evolution. Gratton et al. (1996) were the first to directly compare the $[\mathrm{Fe} / \mathrm{O}]$ abundance ratios of 15 thick disk stars with halo and thin disk populations. Their results reveal a nearly constant ratio in both halo and thick disk, and an increase of $[\mathrm{Fe} / \mathrm{O}]$ by $\sim 0.2$ dex during the transition from the thick to the thin disk population, indicating a sudden decrease in star formation in the solar neighbourhood at that epoch. New, clear evidence of chemical distinction of the thick from thin disk was given in the study of Fuhrmann (1998). For a sample of 50 stars he found a clear separation of the $[\mathrm{Mg} / \mathrm{Fe}]$ ratios between the two stellar populations and inferred an intermediate phase of low or even ceased star formation before the earliest stars of the thin disk were formed $\sim 9$ Gyr ago.

Last year several studies appeared (Gratton et al. 2000; Prochaska et al. 2000; Mashonkina \& Gehren 2000; Bernkopf et al. 2001) which presented strong additional evidence for a distinct chemical history of the thick and thin disk. From the $[\mathrm{Fe} / \mathrm{O}]$ and $[\mathrm{Fe} / \mathrm{Mg}]$ ratios for the sample of about 300 stars Gratton et al. (2000) argue that the halo and thick disk formation occured on a short timescale and that there was a sudden decrease in star formation between the thick and thin disk phases. The new study of the Munich astrophysicists (Bernkopf et al. 2001) for an extended sample of $(\sim 100)$ stars improves the statistical significance of the earlier conclusions of Fuhrmann (1998). For a sample of 10 thick disk stars Prochaska et al. (2000) have determined abundances of several $\alpha$-elements, O, Mg, Si and Ca, light elements, $\mathrm{Na}$ and $\mathrm{Al}$, iron peak elements and heavy elements, $\mathrm{Y}, \mathrm{Ba}$ and Eu. From comparison of their results with abundance studies of the halo, bulge, and thin disk taken from the literature they conclude that in the majority of cases the thick disk stars exhibit $\mathrm{X} / \mathrm{Fe}$ ratios distinct from the thin disk. To derive Eu abundances they used the very weak Eu II subordinate line $\lambda 6645$ and obtained results only for 4 stars. For these stars they found an overabundance of Eu relative to $\mathrm{Ba}$ indicating a dominance of the $r$-process in heavy element production.

In Paper I we have determined the $[\mathrm{Eu} / \mathrm{Ba}]$ abundance ratios for a larger sample (15) of stars on the base of nonlocal thermodynamical equilibrium (NLTE) line formation for BaII and EuII and it was shown that

1. not only the halo but also the thick disk stars reveal an overabundance of europium relative to barium with mean values of $[\mathrm{Eu} / \mathrm{Ba}]=0.64$ and 0.49 , respectively;

2. there is a step-like decrease in the $[\mathrm{Eu} / \mathrm{Ba}]$ ratios at the thick to thin disk transition;

3. the two halo stars with Eu abundances could be determined show an overabundance of europium relative to magnesium of above 0.2 dex.

From these observational results the conclusions important for the Galaxy's evolution follow immediately

- the halo and thick disk stellar populations were formed rapidly during an interval comparable to the evolution time of an AGB progenitor of 3 to $4 M_{\odot}$, because halo and thick disk stars reveal an insignificant contribution of the $s$-process to barium synthesis;

- the star formation rate must have been high at that epoch because during this interval the metallicity of the Galactic gas increased to $[\mathrm{Fe} / \mathrm{H}]=\sim-0.3$;

- before the first stars of the thin disk evolved, there was a phase of nearly ceased star formation during which $r$ process element production stopped but $s$-process nuclei of Ba were synthesized in evolved low-mass stars.

In addition, one problem remained unsolved in Paper I. It is commonly believed that $\mathrm{Mg}$ and Eu are mainly produced in SN II explosions but the question is whether the $\alpha$ - and $r$-process occur in a common site. What are the progenitor masses of SNe II in which the $r$-process runs with the highest efficiency? And what about similar values for $\alpha$-process? Our data on the [Eu/Mg] abundance ratios in the two halo stars point to different sites of $r$-process and $\alpha$-process nucleosynthesis. 
Our work was, however, restricted to a rather small sample of only 15 stars with Eu abundances and 29 stars with Ba abundances obtained. To improve the significance of our earlier conclusions, we extend our analysis in this study to a total of 63 stars. As in Paper I the stars are selected from Fuhrmann's (1998, 2001) lists. Fuhrmann's sample of cool nearby stars with metallicities $[\mathrm{Fe} / \mathrm{H}]$ from -2 to 0.25 includes only main sequence stars (MS) or stars close to the MS with carefully derived stellar parameters $T_{\text {eff }}, \log g,[\mathrm{Fe} / \mathrm{H}]$ and microturbulence $V_{\text {mic }}$ and, thus, provides a reliable base for element abundance determinations and the study of Galactic chemical evolution. For all the stars added to our new sample, highresolution spectra observed at $R \simeq 60000$ are used. We will show in the present analysis that the basic results of Paper I are still valid and some new observational findings constraining the models of Galaxy evolution become apparent. The most important of them is a slight decline of the $[\mathrm{Eu} / \mathrm{Ba}]$ abundance ratios with increasing metallicity for the thick disk stars, which can be used to calculate the duration of star formation in the thick disk stellar population.

In addition to barium and europium, strontium abundances are determined in this study. The most abundant $\mathrm{Sr}$ isotopes, ${ }^{86} \mathrm{Sr},{ }^{87} \mathrm{Sr}$ and ${ }^{88} \mathrm{Sr}$, have masses that lie on the boundary between nuclei produced by the weak and main s-process. Based on calculations of stellar models for 1.5 and $3 M_{\odot}$ AGB stars Arlandini et al. (1999) have found an $85 \%$ contribution of the main $s$-process to solar strontium. The contribution of the weak component can only be estimated: using a classical approach Arlandini et al. obtain $6 \%$, and for the main component $90 \%$; the remaining $4 \%$ correspond to residuals of the $r$-process. In fact, the uncertainty of $r$-process and weak $s$-process contributions may be a factor of 2 or even larger. Keeping in mind that $s$-nuclei of $\mathrm{Ba}$ are produced only by the main $s$-process which is described much better compared with the weak component we can use the strontium-tobarium abundance ratio as a useful diagnostic of the type of $s$-process that formed Sr. Strontium is more abundant than barium and the Sr II resonance lines $\lambda 4077 \AA$ and $\lambda 4215 \AA$ can be detected even in extremely metal-poor stars. However, both lines are strongly blended and element abundances must be derived using synthetic spectra. The study of Gratton \& Sneden (1994) is based on synthetic spectra of only the Sr II $\lambda 4215$ line, but in most investigations (Hartmann \& Gehren 1988; Magain 1989; McWilliam et al. 1995; Ryan et al. 1996) Sr abundances are based on equivalent widths $\left(W_{\lambda}\right)$ of the SrII lines. Other investigations use the weaker Sr I resonance line $\lambda 4607$ (Gratton \& Sneden 1994; Jehin et al. 1999). However, in the case of minor species such as SrI some uncertainties of elemental abundances are expected due to using the LTE assumption. Therefore the observational situation with Sr abundances in cool stars is not clear. In this study we obtain strontium abundances from the Sr II lines on the basis of NLTE line formation using synthetic spectra. NLTE effects for Sr II in cool metal-poor stars are considered for the first time. We use in this study the method of NLTE calculations for Sr II developed by Belyakova \& Mashonkina (1997).

The remaining paper is organized as follows. Observations and stellar parameters are described in Sect. 2. NLTE barium and europium abundances obtained using the methods described in Paper I are discussed in Sect. 3. In Sect. 4 we describe the Sr II model atom and NLTE effects for Sr II. In the next section solar Sr II line profiles are fitted to empirically improve three types of atomic parameters important for further analyses of stellar spectra: the efficiency of collisions with hydrogen atoms in Sr II kinetic equilibrium, van der Waals damping constants of the SrII lines and oscillator strengths of line blends. Stellar strontium NLTE abundances are presented at the end of this section. In the final section we discuss the element abundance ratios and implications for nucleosynthesis and the evolution of the Galaxy.

\section{Observations, stellar parameters and model atmospheres}

Our results are based on spectra observed mostly by Klaus Fuhrmann and in part by Andreas Korn and the late Michael Pfeiffer using the fiber optic Cassegrain échelle spectrograph FOCES fed by the $2.2 \mathrm{~m}$ telescope at the Calar Alto observatory during 10 observing runs in 19952000. The data cover an approximate spectral range of 4000-7000 Å. In total, our sample now includes 63 stars: 27 stars from our previous work (Paper I), 24 stars newly observed in January and May 2000, and 12 thin disk stars observed earlier and added to cover as best as possible the metallicity range of the thin disk. Table 1 lists all the new stars plus 19 stars from Paper I for which Sr abundances were determined. Almost all of the stars were observed at least twice. For the 1995 spectra (11 stars of our old sample) the resolving power was $\sim 40000$ and the later spectra of 52 stars were observed at $\lambda / \Delta \lambda \sim 60000$. The signal-to-noise ratio is $\sim 200$ in the spectral range where the BaII $\lambda 4554, \lambda 5853$ and $\lambda 6496$ lines are located and $\sim 100$ in the range of the Eu II $\lambda 4129$ and Sr II $\lambda 4161, \lambda 4215$ lines.

We use spectra reduced according to the description given in Pfeiffer et al. (1998). Stellar element abundances are derived from line profile fitting and the instrumental profile is found from comparison of FOCES Moon spectra with the Kitt Peak Solar Flux Atlas (Kurucz et al. 1984). Observations are well fitted by a Gaussian of different values for different observing runs (i.e. from $3.2 \mathrm{~km} \mathrm{~s}^{-1}$ to $5 \mathrm{~km} \mathrm{~s}^{-1}$ ).

As in Paper I we use stellar parameters determined mostly by Fuhrmann $(1998,2001)$ spectroscopically: effective temperatures $T_{\text {eff }}$ from Balmer line profile fitting, surface gravities $\log g$ from line wings of the $\mathrm{Mg}$ Ib triplet, metallicities $[\mathrm{Fe} / \mathrm{H}]$ and microturbulence values $V_{\text {mic }}$ from the Fe II line profile fitting. For three stars, HD 19445, BD $2^{\circ} 3375$ and BD $34^{\circ} 2476$, we adopt the stellar parameters determined by Andreas Korn (2000) obtained with 
Table 1. Stellar parameters of the selected sample. Most of the entries are self-explanatory. $V_{\text {mic }}$ is given in $\mathrm{km} \mathrm{s}^{-1}$.

\begin{tabular}{|c|c|c|c|c|c|c|c|}
\hline $\mathrm{HD} / \mathrm{BD}$ & $T_{\text {eff }}$ & $\log g$ & $V_{\text {mic }}$ & {$[\mathrm{Fe} / \mathrm{H}]$} & {$[\mathrm{Ba} / \mathrm{Fe}]$} & $\mathrm{Eu} / \mathrm{Fe}$ & {$[\mathrm{Sr} / \mathrm{Fe}]$} \\
\hline 3795 & 5370 & 3.82 & 1.0 & -0.64 & 0.02 & 0.56 & 0.04 \\
\hline 4614 & 5940 & 4.33 & 1.0 & -0.30 & 0.03 & 0.11 & 0.00 \\
\hline 10519 & 5710 & 4.00 & 1.1 & -0.64 & -0.05 & 0.38 & 0.06 \\
\hline 10697 & 5610 & 3.96 & 1.0 & 0.10 & -0.01 & -0.08 & -0.17 \\
\hline 18757 & 5710 & 4.34 & 1.0 & -0.28 & -0.11 & 0.25 & -0.12 \\
\hline 19445 & 6060 & 4.44 & 1.4 & -1.99 & -0.13 & - & -0.13 \\
\hline 22879 & 5870 & 4.27 & 1.2 & -0.86 & -0.02 & 0.42 & 0.07 \\
\hline 30649 & 5820 & 4.28 & 1.2 & -0.47 & -0.10 & 0.32 & -0.10 \\
\hline 30743 & 6300 & 4.03 & 1.6 & -0.45 & -0.02 & 0.13 & - \\
\hline 37124 & 5610 & 4.44 & 0.9 & -0.44 & -0.12 & 0.30 & -0.11 \\
\hline 43042 & 6440 & 4.23 & 1.5 & 0.04 & 0.00 & -0.02 & 0.10 \\
\hline 45282 & 5280 & 3.12 & 1.4 & -1.52 & -0.07 & 0.60 & -0.08 \\
\hline 52711 & 5890 & 4.31 & 1.0 & -0.16 & 0.01 & 0.05 & -0.02 \\
\hline 55575 & 5890 & 4.25 & 1.0 & -0.36 & -0.05 & 0.20 & -0.10 \\
\hline 58855 & 6310 & 4.16 & 1.4 & -0.32 & 0.08 & - & - \\
\hline 61421 & 6470 & 4.00 & 1.9 & -0.01 & -0.17 & 0.01 & 0.09 \\
\hline 62301 & 5940 & 4.18 & 1.2 & -0.69 & -0.06 & 0.36 & -0.02 \\
\hline 64606 & 5320 & 4.54 & 1.0 & -0.89 & -0.09 & 0.47 & -0.04 \\
\hline 65583 & 5320 & 4.55 & 0.8 & -0.73 & -0.05 & 0.46 & 0.02 \\
\hline 67228 & 5850 & 3.93 & 1.2 & 0.12 & -0.06 & -0.13 & -0.06 \\
\hline 68017 & 5630 & 4.45 & 0.9 & -0.40 & -0.12 & 0.28 & -0.11 \\
\hline 69611 & 5820 & 4.18 & 1.2 & -0.60 & -0.11 & 0.36 & 0.05 \\
\hline 84937 & 6350 & 4.03 & 1.7 & -2.07 & 0.00 & - & -0.12 \\
\hline 90508 & 5800 & 4.35 & 1.0 & -0.33 & -0.03 & 0.26 & -0.12 \\
\hline 102158 & 5760 & 4.24 & 1.1 & -0.46 & -0.13 & 0.34 & -0.01 \\
\hline 103095 & 5110 & 4.66 & 0.8 & -1.35 & 0.00 & 0.55 & -0.11 \\
\hline 109358 & 5860 & 4.36 & 1.1 & -0.21 & -0.07 & - & - \\
\hline 112758 & 5240 & 4.62 & 0.7 & -0.43 & -0.13 & 0.28 & -0.12 \\
\hline 114710 & 6000 & 4.30 & 1.1 & -0.03 & 0.07 & 0.01 & 0.05 \\
\hline 117176 & 5480 & 3.83 & 1.0 & -0.11 & -0.04 & 0.04 & -0.14 \\
\hline 121560 & 6140 & 4.27 & 1.2 & -0.43 & 0.06 & 0.14 & 0.08 \\
\hline 126053 & 5690 & 4.45 & 1.0 & -0.35 & -0.11 & 0.14 & -0.10 \\
\hline 130322 & 5390 & 4.55 & 0.8 & 0.04 & 0.03 & -0.04 & - \\
\hline 132142 & 5240 & 4.58 & 0.7 & -0.39 & -0.09 & 0.26 & - \\
\hline 134987 & 5740 & 4.25 & 1.0 & 0.25 & -0.12 & -0.17 & -0.10 \\
\hline 142373 & 5840 & 3.84 & 1.2 & -0.57 & -0.06 & 0.23 & -0.06 \\
\hline 144579 & 5330 & 4.59 & 0.8 & -0.69 & -0.08 & 0.46 & -0.04 \\
\hline 157214 & 5735 & 4.24 & 1.0 & -0.34 & -0.13 & 0.34 & -0.08 \\
\hline 168009 & 5785 & 4.23 & 1.0 & -0.03 & -0.08 & -0.08 & -0.09 \\
\hline 176377 & 5860 & 4.43 & 0.9 & -0.27 & 0.14 & - & - \\
\hline 179957 & 5740 & 4.38 & 0.9 & -0.01 & -0.06 & 0.03 & -0.14 \\
\hline 179958 & 5760 & 4.32 & 0.9 & 0.02 & -0.04 & 0.00 & -0.12 \\
\hline 187923 & 5730 & 4.01 & 1.1 & -0.17 & -0.05 & 0.13 & -0.10 \\
\hline 188512 & 5110 & 3.60 & 0.9 & -0.17 & 0.09 & 0.03 & -0.10 \\
\hline 194598 & 6060 & 4.27 & 1.4 & -1.12 & -0.03 & 0.58 & -0.11 \\
\hline 195019 & 5800 & 4.16 & 1.0 & 0.04 & -0.03 & 0.00 & -0.12 \\
\hline 198149 & 4990 & 3.40 & 1.0 & -0.14 & 0.04 & 0.01 & - \\
\hline 201891 & 5940 & 4.24 & 1.2 & -1.05 & -0.05 & 0.42 & -0.02 \\
\hline 207978 & 6310 & 3.94 & 1.6 & -0.52 & 0.00 & - & - \\
\hline 209458 & 6080 & 4.33 & 1.1 & -0.06 & 0.10 & 0.10 & 0.11 \\
\hline 222794 & 5620 & 3.94 & 1.2 & -0.69 & -0.10 & 0.38 & 0.05 \\
\hline $2^{\circ} 3375$ & 6200 & 4.31 & 1.4 & -2.15 & -0.18 & - & -0.06 \\
\hline
\end{tabular}

\begin{tabular}{rrrrrrrr}
\hline $\mathrm{HD} / \mathrm{BD}$ & $T_{\text {eff }}$ & $\log g$ & $V_{\text {mic }}$ & {$[\mathrm{Fe} / \mathrm{H}]$} & {$[\mathrm{Ba} / \mathrm{Fe}]$} & {$[\mathrm{Eu} / \mathrm{Fe}]$} & {$[\mathrm{Sr} / \mathrm{Fe}]$} \\
\hline $0^{\circ} 2245$ & 5630 & 3.85 & 1.2 & -1.13 & 0.19 & - & 0.22 \\
$34^{\circ} 2476$ & 6330 & 4.03 & 1.8 & -1.96 & 0.13 & - & -0.21 \\
$66^{\circ} 268$ & 5340 & 4.60 & 0.9 & -2.20 & 0.07 & - & -0.11 \\
\hline
\end{tabular}

the same methods. All parameters are given in Table 1. The identification of the stellar population for all stars of our sample is from Fuhrmann (1998) and Bernkopf et al. (2001), based on the star's kinematics, $\alpha$-element enhancement and age.

For each star a line-blanketed LTE model atmosphere has been generated at given values of $T_{\text {eff }}, \log g,[\mathrm{Fe} / \mathrm{H}]$ and $[\alpha / \mathrm{Fe}]$, where $[\alpha / \mathrm{Fe}]$ is the relative abundance of the most abundant $\alpha$-process elements $\mathrm{O}, \mathrm{Mg}$ and $\mathrm{Si}$, which in cool stellar atmospheres contribute in significant amounts to the electron pressure. We assume that oxygen and silicon abundances follow magnesium and adopt $[\alpha / \mathrm{Fe}]=$ $[\mathrm{Mg} / \mathrm{Fe}]$. The $[\mathrm{Mg} / \mathrm{Fe}]$ abundance ratios are taken from Fuhrmann (1998) and Bernkopf et al. (2001) analyses. Three aspects concerning model atmosphere calculations are worth mentioning

1. The mixing-length parameter $l / H_{\mathrm{p}}$ was adopted to be 0.5 ;

2. The opacity distribution functions (ODF) are interpolated from Kurucz' (1994) ODF tables for the proper stellar metallicities. In addition, they were scaled by -0.16 dex to reset the iron opacity calculated by Kurucz with $\log \varepsilon_{\mathrm{Fe}}=7.67$ to the improved meteoritic value $\log \varepsilon_{\mathrm{Fe}}=7.51$, which we believe to be the best representation of the solar mixture. We refer to abundances on the usual scale where $\log \varepsilon_{\mathrm{H}}=12$;

3 . The $b-f$ opacities were computed with solar abundances taken from Holweger (1979) and scaled according to the stellar metallicity. In addition, abundances of $\alpha$-elements $\mathrm{O}, \mathrm{Mg}$ and $\mathrm{Si}$ were scaled by the stellar $\mathrm{Mg} / \mathrm{Fe}$ ratio. We note that the additional electron pressure in $\alpha$-enhanced models results in a measurable weakening of spectral lines of dominant ionization stages such as Sr II, BaII, Eu II. The effect increases with decreasing temperature. For example, an increase of $\alpha$-element abundances by 0.4 dex in a model atmosphere leads to Ba abundances obtained from stellar spectra increasing by about 0.1 dex.

In Paper I for stars with $[\mathrm{Fe} / \mathrm{H}] \leq-0.6$, model atmospheres were interpolated from a grid of $\alpha$-enhanced models with $[\alpha / \mathrm{Fe}]=0.4$, whereas those for thin disk stars referred to a grid of model atmospheres without any $\alpha$ enhancement. However, the observed $[\mathrm{Mg} / \mathrm{Fe}]$ ratio can differ from 0.4 dex in metal-poor stars (for example, HD 103095: $[\mathrm{Mg} / \mathrm{Fe}]=0.28)$ and from 0 in thin disk stars (for example, HD 117176: $[\mathrm{Mg} / \mathrm{Fe}]=0.08$ ). For this reason Ba abundances obtained in our present study may deviate by up to 0.04 dex from the corresponding values calculated in Paper I.

\section{Barium and europium abundances for the stars}

We use the same method as in Paper I to derive Ba and Eu abundances for the stars. The synthetic line profiles are computed using the departure coefficients of the Ba II and Eu II levels from the code NONLTE3 (Sakhibullin 1983) and the LTE assumption for other atoms. The line list 
is extracted from Kurucz' (1994) compilation, and it includes all the relevant atomic and molecular lines. A differential analysis with respect to the Sun is performed. Solar barium and europium abundances, $\log \varepsilon_{\mathrm{Ba}, \odot}=2.21$ and $\log \varepsilon_{\mathrm{Eu}, \odot}=0.53$, and van der Waals damping constants $C_{6}$ for the Ba II and Eu II lines were determined in Paper I from solar line profile fitting. The methods of NLTE calculations for BaII and Eu II were developed earlier (Mashonkina \& Bikmaev 1996; Mashonkina et al. 1999; Mashonkina 2000; Paper I). Some examples of the Ba II and Eu II stellar line profile fitting were given in Paper I.

Barium abundances have now been determined for the 63 stars most of which are listed in Table 1; for 62 of them abundances are obtained both from the subordinate Ba II lines, $\lambda 5853$ and $\lambda 6496$, and from the resonance line $\lambda 4554$. As discussed earlier the Ba II resonance line is strongly affected by hyperfine structure (HFS) and, as a result, Ba abundances derived from this line depend on the even-toodd $\mathrm{Ba}$ isotope abundance ratio adopted in calculations. A difference between $\mathrm{Ba}$ abundances obtained at solar ratio 82:18 (Cameron 1982) and the pure $r$-process ratio 56:44 (Arlandini et al. 1999) can reach to 0.2 dex (HD 45282) and it is minimum (0.08 dex) for the most metal-poor stars of our sample. That is why we prefer to use Ba abundances from the subordinate lines free of HFS effect. However, for the three most metal-poor stars with $[\mathrm{Fe} / \mathrm{H}]<-2$ the only subordinate line available, $\lambda 6496$, is very weak, and the $\mathrm{Ba}$ abundance is determined with an uncertainty of about 0.1 dex. For these stars the resonance line profile leads to a much better fit, and the Ba abundance obtained is more reliable, provided that a realistic even-to-odd $\mathrm{Ba}$ isotopic ratio is used. We have shown in Paper I that barium seen in halo stars must have been mainly produced by the $r$-process. Assuming a pure $r$-process we have found Ba abundances from the $\lambda 4554$ line and compared them with the abundances from the $\lambda 6496$ line:

$\begin{array}{lcc} & {\left[\mathrm{Ba}(\lambda 4554)_{r} / \mathrm{Fe}\right]} & {[\mathrm{Ba}(\lambda 6496) / \mathrm{Fe}]} \\ \mathrm{HD} 84937 & -0.02 & 0.00 \\ \mathrm{BD} \mathrm{2} 3375 & -0.11 & -0.18 \\ \mathrm{BD} 66^{\circ} 268 & 0.01 & 0.07 .\end{array}$

For each star the difference $\log \varepsilon(\lambda 4554)_{r}-\log \varepsilon(\lambda 6496)$ is within the $\mathrm{Ba}$ abundance errors, and keeping in mind that the abundance from the resonance line is more reliable we adopt it as the final $\mathrm{Ba}$ abundance. Thus, for these three stars and for BD $34^{\circ} 2476$, with only the resonance line available, Ba abundances have been obtained from the resonance line under the assumption of a pure $r$-process even-to-odd $\mathrm{Ba}$ isotope ratio. For the remaining 59 stars we have obtained $\mathrm{Ba}$ abundances from the subordinate lines. If both of them were available the average value was calculated.

NLTE effects for BaII in cool stars were described in detail earlier (Mashonkina et al. 1999). Here we just note that the kinetic equilibrium of BaII is strongly affected by radiative processes in $b-b$ transitions because this is the dominant ionization stage. As a consequence NLTE
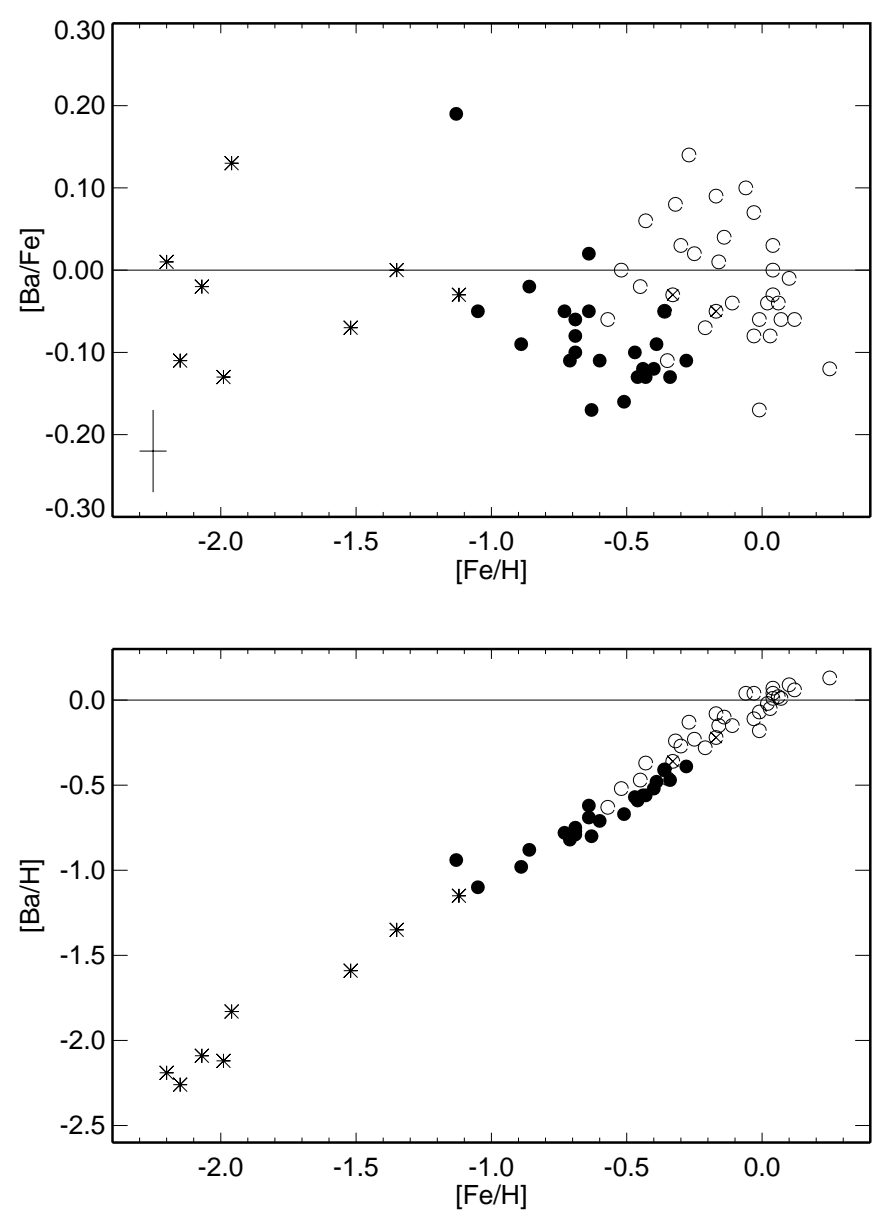

Fig. 1. The runs of $[\mathrm{Ba} / \mathrm{Fe}]$ and $[\mathrm{Ba} / \mathrm{H}]$ with $[\mathrm{Fe} / \mathrm{H}]$. Symbols correspond to the thin disk (open circles), the thick disk (filled circles), and the halo stars (asterisks). The two stars indicated by a cross in an open circle are transition stars according to Fuhrmann (1998). Error bars are indicated at the lower left.

effects for Ba II depend on the Ba abundance which correlates with the general metallicity of the model atmosphere. Thus, NLTE leads to a strengthening of the BaII lines compared with the LTE case at $[\mathrm{M} / \mathrm{H}]>-1.9$ and to the opposite effect at lower metallicities. NLTE effects are small for the weakest line $\lambda 5853$. NLTE abundance corrections $\Delta_{\mathrm{NLTE}}=\log \varepsilon_{\mathrm{NLTE}}-\log \varepsilon_{\mathrm{LTE}}$ do not exceed 0.1 dex by absolute value. Significant NLTE effects have been found for the second subordinate line 16496: $\Delta_{\mathrm{NLTE}}$ is -0.2 dex on average in the metallicity range $-1<[\mathrm{Fe} / \mathrm{H}]<0.25$; it reduces by absolute value to $0.10-0.15$ dex at metallicities between -1.5 and -1 , and it becomes positive up to 0.15 dex at even lower $[\mathrm{Fe} / \mathrm{H}] . \Delta_{\mathrm{NLTE}}$ is positive also for the resonance line in the four halo stars with $\mathrm{Ba}$ abundances derived from this line. Its value is at maximum for the hottest star, HD 84937: $\Delta_{\mathrm{NLTE}}=0.30$ dex and smaller for the coolest star, BD $66^{\circ} 268: \Delta_{\mathrm{NLTE}}=0.08$ dex. For 52 stars with both subordinate lines available the mean value of the difference between NLTE abundances derived from $\lambda 6496$ and $\lambda 5853$ equals $0.00 \pm 0.03 \mathrm{dex}$, while under the LTE assumption Ba abundances from the first line are 

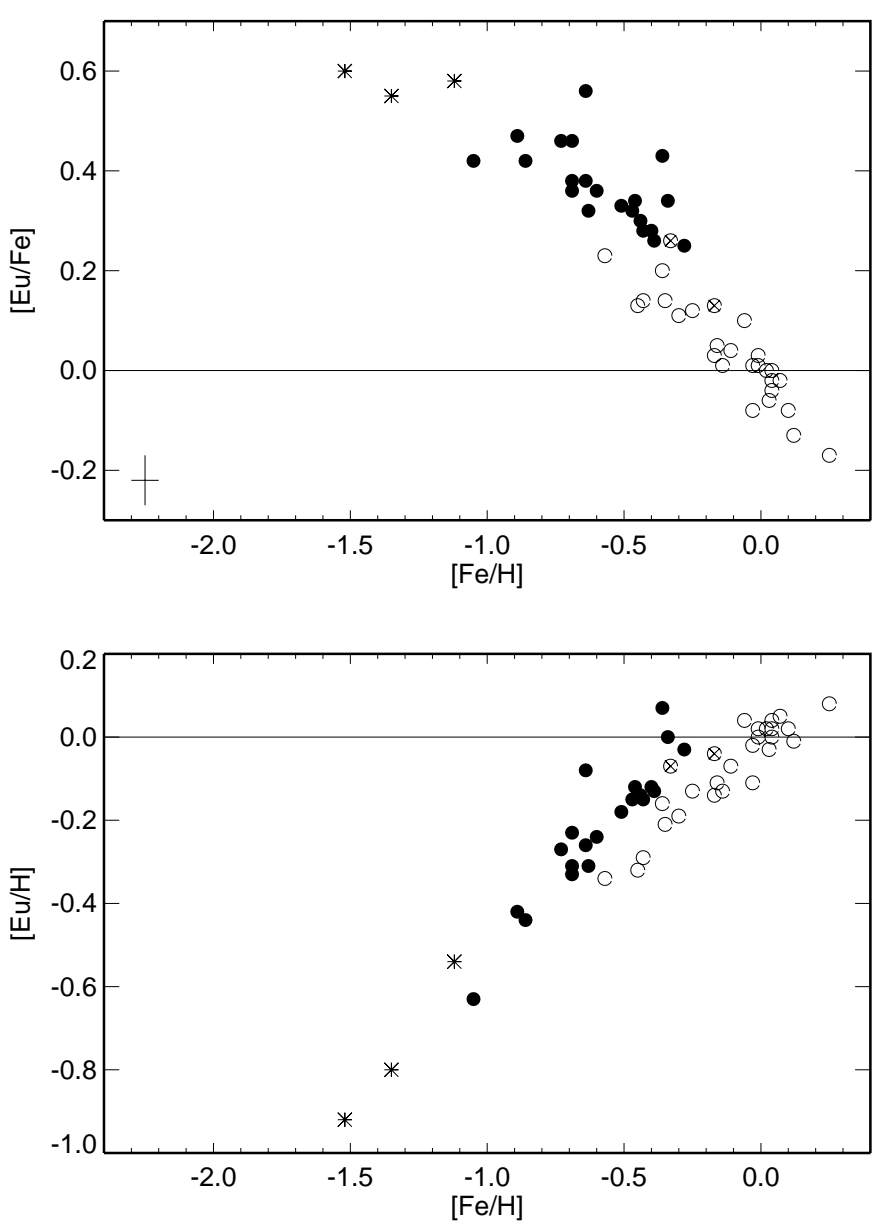

Fig. 2. Variation of $[\mathrm{Eu} / \mathrm{Fe}]$ (top panel) and $[\mathrm{Eu} / \mathrm{H}]$ (bottom panel) with $[\mathrm{Fe} / \mathrm{H}]$. Symbols are the same as in Fig. 1.

systematically overestimated relative to $\log \varepsilon_{\mathrm{LTE}}(\lambda 5853)$ with the mean difference of $0.11 \pm 0.04$. This gives reason to believe that the uncertainty of our NLTE line formation treatment leads to $\mathrm{Ba}$ abundance errors not greater than 0.03 dex.

The final $[\mathrm{Ba} / \mathrm{Fe}]$ abundance ratios are presented in Table 1 and Fig. 1. In addition, in the bottom panel of Fig. 1 we give $[\mathrm{Ba} / \mathrm{H}]$. As the reference solar abundance $\log \varepsilon_{\mathrm{Fe}, \odot}=7.51$ adopted by Fuhrmann $(1998,2001)$ is used in stellar metallicity determinations. As discussed in Paper I, uncertainties of stellar parameters cause abundance errors up to $\Delta \log \varepsilon_{\mathrm{Ba}}=0.11$ dex.

Europium abundances have been derived from the Eu II $\lambda 4129$ line for 51 stars of our sample. Even at spectral resolving power $R=60000$ this line can not be extracted from noise in spectra of the hot halo stars HD 19445, HD 84937, BD $2^{\circ} 3375$ and $\mathrm{BD} 34^{\circ} 2476$. For the cool star $\mathrm{BD} 66^{\circ} 268$, with the lowest metallicity, $[\mathrm{Fe} / \mathrm{H}]=-2.20$, only a spectrum observed at $R=40000$ is available, and though the Eu II $\lambda 4129$ line is detected its profile cannot be satisfactorily fitted. In addition, Eu abundances could not be determined for all the stars observed in May 1997 and September 1996 because no spectra were reduced shortward of $4300 \AA$.
Table 2. Mean values $\overline{[\mathrm{Ba} / \mathrm{Fe}]}$ for thin disk stars of different ages.

\begin{tabular}{ccc}
\hline Age $[\mathrm{Gyr}]$ & Number of stars & $\overline{[\mathrm{Ba} / \mathrm{Fe}]}$ \\
\hline$>8$ & 7 & $-0.06 \pm 0.03$ \\
$6-8$ & 7 & $-0.03 \pm 0.04$ \\
$4-6$ & 8 & $0.01 \pm 0.07$ \\
$2-4$ & 5 & $0.06 \pm 0.07$ \\
$\leq 2$ & 3 & $?$ \\
\hline
\end{tabular}

As discussed in Paper I NLTE effects weaken the Eu II $\lambda 4129$ line compared with the LTE case and NLTE abundance corrections are positive. For our stars $\Delta_{\mathrm{NLTE}}$ ranges from 0.03 dex to 0.07 dex. The final $[\mathrm{Eu} / \mathrm{Fe}] \mathrm{ra}-$ tios are presented in Table 1 and Fig. 2, where the run of $[\mathrm{Eu} / \mathrm{H}]$ with metallicity is shown, too. Uncertainties of stellar parameters cause abundance errors up to $\Delta \log \varepsilon_{\mathrm{Eu}}=0.06 \operatorname{dex}$ (Paper I). The Eu II $\lambda 4129$ line is located in a crowded spectral range and this can lead to additional errors. To find a continuum level we fitted observed spectra in the spectral range from $4123 \AA$ to $4135 \AA$. The difference of Eu abundances derived from two spectra of a star is usually within 0.05 dex. We estimate a total Eu abundance error of 0.1 dex.

Figures 1 and 2 confirm the results obtained in Paper I and show new features that have become apparent due to the extension of the stellar sample. We summarize them as follows

- In the halo stars the $[\mathrm{Ba} / \mathrm{Fe}]$ ratios are mainly between 0.01 and -0.13 with the exception, BD $34^{\circ} 2476$, where barium is evidently overabundant relative to iron: $[\mathrm{Ba} / \mathrm{Fe}]=0.13$. The three halo stars with Eu abundances available show $[\mathrm{Eu} / \mathrm{Fe}]$ ratios that are close together with a mean value of 0.58 ;

- The thick disk stars reveal a $\mathrm{Ba}$ underabundance relative to iron with a mean value $[\mathrm{Ba} / \mathrm{Fe}]=-0.10 \pm$ 0.04 (except for $\mathrm{BD} 0^{\circ} 2245$ with $[\mathrm{Ba} / \mathrm{Fe}]=0.19$; we will return to this star below when discussing other element abundance ratios). A slight decline of the $[\mathrm{Ba} / \mathrm{Fe}]$ abundance ratios with increasing metallicity should be noted. In contrast to Ba, europium is overabundant relative to iron in thick disk stars with a clear decline of the $[\mathrm{Eu} / \mathrm{Fe}]$ abundance ratios from about 0.5 at $[\mathrm{Fe} / \mathrm{H}]=-0.9$ to 0.25 at $[\mathrm{Fe} / \mathrm{H}]=-0.28$;

- The thin disk stars reveal, on average, solar $[\mathrm{Ba} / \mathrm{Fe}]$ abundance ratios with a mean value of $\overline{[\mathrm{Ba} / \mathrm{Fe}]}=$ $-0.01 \pm 0.06$ and a decline of the $[\mathrm{Eu} / \mathrm{Fe}]$ abundance ratios with increasing metallicity. There is a rather large spread in $[\mathrm{Ba} / \mathrm{Fe}]$ up to 0.3 dex that exceeds Ba abundance errors. At the same time, a spread in $[\mathrm{Eu} / \mathrm{Fe}]$ is within abundance errors;

- We emphasize that both $[\mathrm{Ba} / \mathrm{Fe}]$ and $[\mathrm{Eu} / \mathrm{Fe}]$ abundance ratios reveal a distinction between thick and thin disk stellar populations. This distinction is more evident in the runs of $[\mathrm{Ba} / \mathrm{H}]$ and $[\mathrm{Eu} / \mathrm{H}]$ along metallicity (Figs. 1 and 2, bottom panels). In the region of overlapping metallicities $\mathrm{Ba}$ abundances in the thick 


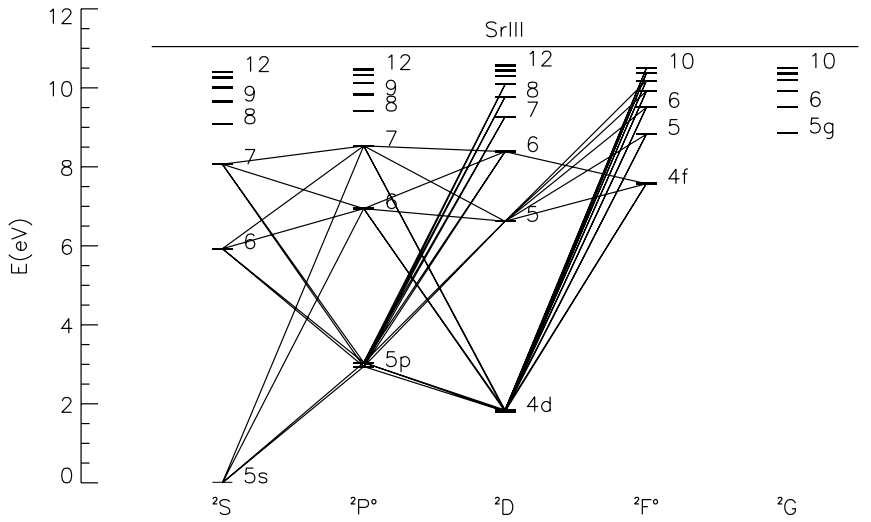

Fig. 3. The Sr model atom. Linearized transitions are shown as solid lines.

disk stars are lower by 0.1 dex, on average, while Eu abundances are higher by 0.15 dex compared with the thin disk stars.

We note the star HD 3795 which reveals a chemical composition closer to the halo than to the thick disk population. From the two high-quality spectra observed at $R \simeq 60000$ we have determined the elemental abundances:

$[\mathrm{Ba} / \mathrm{Fe}]=0.02$ and 0.01 from the Ba II $\lambda 5853$ and $\lambda 6496$, $[\mathrm{Eu} / \mathrm{Fe}]=0.56$ and 0.61 from the Eu II $\lambda 4129$ and $\lambda 6645$. The ratios $[\mathrm{Ba} / \mathrm{Fe}],[\mathrm{Eu} / \mathrm{Fe}]$ and $[\mathrm{Eu} / \mathrm{Mg}]$ in this star are higher by $0.12-0.17$ dex compared with the corresponding values in other thick disk stars.

We have tried to find the reason for the large spread of $[\mathrm{Ba} / \mathrm{Fe}]$ among the thin disk stars and noted a marginal correlation between the $[\mathrm{Ba} / \mathrm{Fe}]$ abundance ratio and star's age. Stellar ages have been estimated by Bernkopf et al. (2001) using evolutionary tracks of VandenBerg et al. (2000) and recent own calculations. Allowing for an uncertainty of 1 Gyr for the stellar age estimates we combined the stars into separate age groups and calculated for each group the mean value $\overline{[\mathrm{Ba} / \mathrm{Fe}]}$ (Table 2 ). We do not give $\overline{[\mathrm{Ba} / \mathrm{Fe}]}$ for stars younger than $2 \mathrm{Gyr}$ because the three stars available do not represent this group in a statistically reliable way; for two of them, HD 43042 and HD 130322, $[\mathrm{Ba} / \mathrm{Fe}]=0.00$ and 0.03 , respectively, and we note a surprisingly low $[\mathrm{Ba} / \mathrm{Fe}]$ abundance ratio $(-0.17)$ for Procyon (HD 61421). It is evident from Table 2 that during thin disk evolution the $\mathrm{Ba}$ abundance in interstellar matter increased relative to the iron abundance by about 0.12 dex. So, at least part of the observed spread in $[\mathrm{Ba} / \mathrm{Fe}]$ may not be random. At the same time, Edvardsson et al. (1993) first noted, and Fuhrmann (2001) confirmed later, that the metallicity of thin disk stars correlates only weakly with the stellar age. For this reason we do not see any regular behaviour of the $[\mathrm{Ba} / \mathrm{Fe}]$ ratios plotted against $[\mathrm{Fe} / \mathrm{H}]$ of thin disk stars (Fig. 1).

\section{NLTE calculations for Sr II}

The NLTE problem for Sr II was first treated on the base of a realistic model atom by Belyakova \& Mashonkina (1997). Here we describe briefly the atomic data and new results.

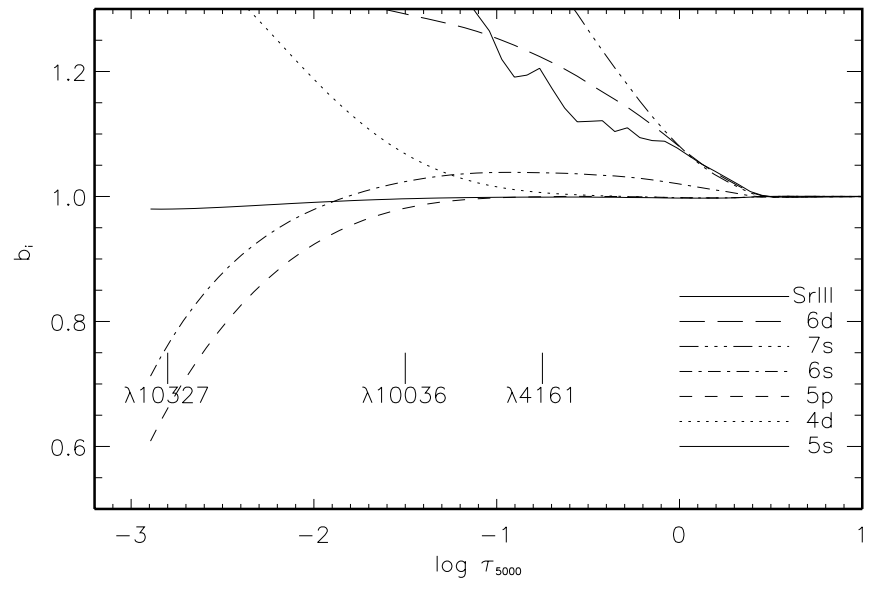

Fig. 4. Departure coefficients $b_{i}$ for some levels of Sr II in the model atmosphere of the Sun. Tick marks indicate the locations of line center optical depth unity for the Sr II lines. The resonance line core forms above $\log \tau_{5000}=-4$.

The Sr II model atom contains all levels with $n \leq 12$ and $l \leq 4$. Doublet fine structure is neglected except for the $4 \mathrm{~d}^{2} \mathrm{D}$ and $5 \mathrm{p}^{2} \mathrm{P}^{\circ}$ splitting. Thus, 40 bound levels of SrII and the ground state of Sr III are included in the model atom. The corresponding Grotrian diagram is shown in Fig. 3. The Sr I levels are taken into account only for number conservation because in all stellar atmospheres considered the ratio $n(\mathrm{Sr} \mathrm{I}) / n(\mathrm{Sr} \mathrm{II})$ is smaller than $10^{-4}$ due to the low ionization energy of Sr I: $\chi($ Sr I $)=5.695 \mathrm{eV}$.

The energy levels are from Moore (1952) and Lindgard \& Nielsen (1977). Sr II transition probabilities from Wiese \& Martin (1980) are believed to be the best. If they are not available the data of Kurucz (1994) or Lindgard \& Nielsen (1977) are taken giving preference to the first of the two sources. Photoionization cross-sections for $n \mathrm{~s}, n \mathrm{p}$ and $n \mathrm{~d}$ levels have been calculated by the quantum defect method using Peach's (1967) tables. For the remaining levels hydrogenic cross-sections are computed. For electron impact excitation we use the formula of van Regemorter (1962) for allowed transitions and that of Allen (1973) for forbidden ones. Electron impact ionization cross-sections are computed according to Drawin (1961). For hydrogen collisions, we use the formula of Steenbock \& Holweger (1984). Since this formula provides only an order of magnitude estimate, the cross-sections were multiplied by appropriate scaling factors in order to produce the best fit to the solar Sr II line profiles.

The Sr II kinetic equilibrium is calculated using the code NONLTE3 (Sakhibullin 1983), which is based on the complete linearization method as described by Auer \& Heasley (1976). The advanced method of calculations has been described in detail in our previous work (Mashonkina et al. 1999).

The Sr II term structure is similar to that of BaII, and the same mechanisms of departures from LTE are responsible for both ions. NLTE effects for Ba II were described 
in detail earlier (Mashonkina et al. 1999). In Fig. 4 the departure coefficients, $b_{i}$, are shown for the solar atmosphere as a function of continuum optical depth $\tau_{5000}$ at $\lambda=5000 \AA$. In the first place, we are interested in the behaviour of the levels contributing to the subsequent line profile synthesis. These are the $5 \mathrm{~s}, 4 \mathrm{~d}, 5 \mathrm{p}$ and $6 \mathrm{~s}$ levels.

As Sr II is the dominant ionization stage, no process affects the ground state population, and 5s keeps its thermodynamic equilibrium value. The metastable level $4 \mathrm{~d}$ is separated by $1.8 \mathrm{eV}$ from the ground state and by $1.14 \mathrm{eV}$ from $5 \mathrm{p}$, and therefore collisional and radiative transitions $4 \mathrm{~d}-5 \mathrm{p}$ have stronger effects on the $4 \mathrm{~d}$ level population compared with collisional coupling of this level to the ground state. The departure coefficients of $4 \mathrm{~d}$ and $5 \mathrm{p}$ begin to deviate from 1 at the depths around $\log \tau_{5000}=$ -1 where photon losses in the weakest line $\lambda 10036$ of the multiplet $4 \mathrm{~d}-5 \mathrm{p}$ start to become important. The $4 \mathrm{~d}$ overpopulation and $5 \mathrm{p}$ underpopulation are amplified in the upper layers which are transparent with respect to the radiation of the two strong lines of that multiplet. The overpopulation outside $\log \tau_{5000}=0.4$ of all levels above $6 \mathrm{~s}$ is due to line pumping. Inside $\log \tau_{5000} \simeq-1.5$ the 5 p-level follows the ground state due to strong radiative and collisional coupling. Several transitions such as $5 \mathrm{p} \rightarrow 6 \mathrm{~d}, 5 \mathrm{p} \rightarrow 7 \mathrm{~s}, 4 \mathrm{~d} \rightarrow 4 \mathrm{f}$ are pumped by $J_{\nu}-B_{\nu}\left(T_{\mathrm{e}}\right)$ excess radiation in the layers where the line wing optical depth drops below 1 .

From this behaviour of departure coefficients we expect that the Sr II resonance lines $\lambda 4077, \lambda 4215$, and the lines $\lambda 10036, \lambda 10327, \lambda 10914$ of multiplet $4 d-5 p$ are amplified, whereas $\lambda 4161$ arising from $5 \mathrm{p}$ is weakened compared with the LTE case. In line formation layers the departure coefficients of the lower levels of $5 s-5 p$ and $5 p-6 s$ transitions equal 1, and NLTE effects for the resonance line and $\lambda 4161$ are caused by a deviation of the source function $S_{\mathrm{lu}}$ from $B_{\nu}\left(T_{\mathrm{e}}\right): S_{5 \mathrm{~s}, 5 \mathrm{p}} \simeq b_{5 \mathrm{p}} / b_{5 \mathrm{~s}} B_{\nu}\left(T_{\mathrm{e}}\right)<B_{\nu}\left(T_{\mathrm{e}}\right)$ for the resonance lines and $S_{5 \mathrm{p}, 6 \mathrm{~s}} \simeq b(6 \mathrm{~s}) / b(5 \mathrm{p}) B_{\nu}\left(T_{\mathrm{e}}\right)>B_{\nu}\left(T_{\mathrm{e}}\right)$ for $\lambda 4161$. For the infrared triplet lines both $b_{1}>1$ and $b_{\mathrm{u}} / b_{1}<1$ are valid in line formation layers resulting in much larger NLTE effects compared with the resonance lines and $\lambda 4161$ : for the Sun the NLTE abundance correction $\Delta_{\text {NLTE }}$ is between -0.03 and -0.01 dex for $\lambda 4215$ and between 0.02 and 0.03 dex for $\lambda 4161$ depending on the efficiency of $\mathrm{H}$ atom collisions while $\Delta_{\mathrm{NLTE}}$ ranges from -0.18 to -0.35 dex for $\lambda 10327$.

A similar behaviour of the departure coefficients resulting in an amplification of the $\lambda 4215$ line and a weakening of $\lambda 4161$ has been found for all the stars of our sample. The first NLTE calculations for Sr II (Belyakova \& Mashonkina 1997) have shown that in very metal-poor atmospheres the Sr II resonance lines are not strengthened but weakened compared with the LTE case. The same phenomenon was found for the BaII lines, too (see Sect. 3). For the Ba II lines $\Delta_{\mathrm{NLTE}}$ changes its sign at $[\mathrm{Fe} / \mathrm{H}]$ between -1.5 and -1.9 depending on $T_{\text {eff }}$ while for the $\mathrm{Sr}$ II lines such a transition range is shifted to lower metallicities between -2.1 and -3.0 . At $[\mathrm{Fe} / \mathrm{H}]=-2.5$ NLTE effects for the Sr II resonance lines depend strongly on $T_{\text {eff }}$,
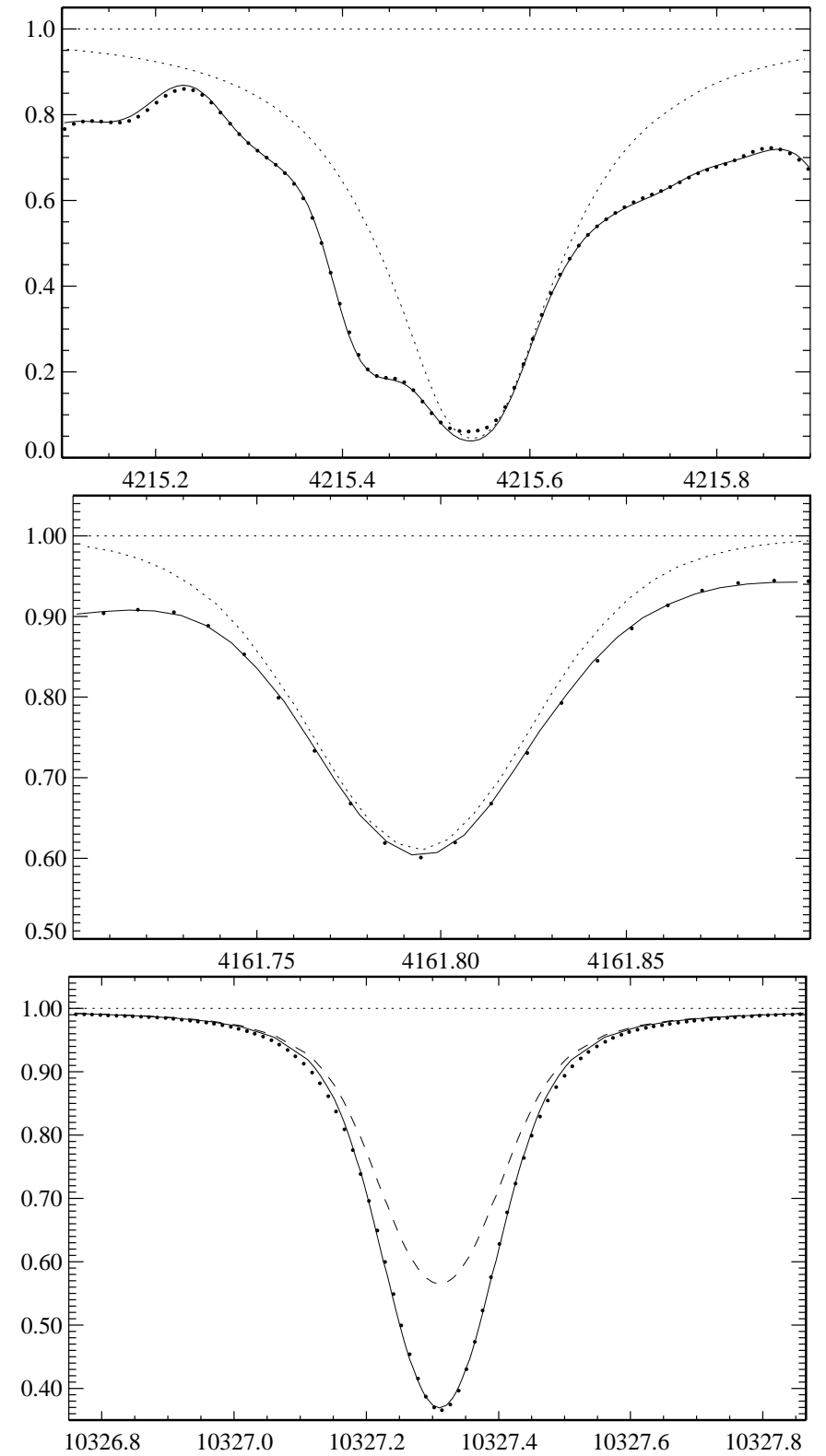

Fig. 5. Synthetic NLTE (continuous line) flux profiles of the Sr II lines compared with the observed spectrum of the Kurucz et al. (1984) solar flux atlas (bold dots). The pure Sr II $\lambda 4215$ and $\lambda 4161$ NLTE profiles (dotted line) are shown for comparison. For the Sr II $\lambda 10327$ the LTE profile is given as dashed line. See text for discussion of the fitting parameters.

$\log g$ and $[\mathrm{Fe} / \mathrm{H}]$, and neglecting NLTE effects can lead to strontium abundance errors up to 1 dex (Belyakova \& Mashonkina 1997).

\section{Strontium abundances}

\subsection{Solar Sr II lines}

The Sr II resonance line $\lambda 4215$ and the subordinate line $\lambda 4161$ are used in this study to determine stellar Sr abundances. Both of them are blended. Solar profiles of these lines are fitted to improve atomic parameters of blending 
lines. Another kind of important atomic data is the efficiency of hydrogen collisions in the Sr II kinetic equilibrium calculations which is represented by a scaling factor $k_{\mathrm{H}}$ applied to Steenbock \& Holweger's (1984) version of Drawin's $(1968,1969)$ formula for the computation of $\mathrm{H}$ atom collisional rates. As mentioned above, the lines $\lambda 10327$ and $\lambda 10914$ reveal strong NLTE effects, and they are therefore most suitable to estimate this scaling factor from solar line profile fitting.

We use solar flux observations taken from the Kitt Peak Solar Atlas (Kurucz et al. 1984). Our synthetic flux profiles are convolved with a profile that combines a rotational broadening of $1.8 \mathrm{~km} \mathrm{~s}^{-1}$ and broadening by macroturbulence with a radial-tangential profile of $V_{\mathrm{mac}}=$ $2.8 \mathrm{~km} \mathrm{~s}^{-1}$ for the infrared lines, $V_{\text {mac }}=3.2 \mathrm{~km} \mathrm{~s}^{-1}$ for $\lambda 4215$ and $V_{\text {mac }}=3.5 \mathrm{kms}^{-1}$ for $\lambda 4161$. For the solar $\mathrm{Sr}$ abundance we accept the meteoritic value $\log \varepsilon_{\mathrm{Sr}}=2.92$ from Grevesse et al. (1996). A depth-independent microturbulence of $0.8 \mathrm{~km} \mathrm{~s}^{-1}$ is adopted. For a calculation of van der Waals damping constants $C_{6}$ we have derived a formula based on Anstee \& O'Mara's (1995) calculations, where

$\log C_{6}=-42.598+15.13 \alpha+(1-2.5 \alpha) \log v+2.5 \log \Upsilon$,

with

$\Upsilon=\left(\frac{\sigma_{0}}{\pi} \Gamma\left(\frac{4-\alpha}{2}\right)\right)$.

Here $v=(8 k T / \pi \mu)^{1 / 2}$ and $\mu=M_{\mathrm{A}} M_{\mathrm{H}} /\left(M_{\mathrm{A}}+M_{\mathrm{H}}\right)$ is the reduced mass; $\sigma_{0}$ is a line-broadening cross-section and $\alpha$ is a velocity parameter. The values of $\sigma_{0}$ and $\alpha$ are tabulated in the papers of Anstee \& O'Mara (1995), Barklem \& O'Mara $(1997,2000)$, and Barklem et al. (1998). The $C_{6^{-}}$ value depends only slightly on temperature as $T^{0.5-1.25 \alpha}$. At typical values of $\alpha$ (between 0.2 and 0.4 ) $\log C_{6}$ changes by 0.04 at maximum as one goes from $T=4000 \mathrm{~K}$ to $T=6000 \mathrm{~K}$. Therefore the small variation of $C_{6}$ across line formation layers can be neglected in calculations of line profiles, and we use in this study depth-independent $C_{6}$-values computed at $T=6000 \mathrm{~K}$.

$\boldsymbol{\lambda 1 0 3 2 7}$ and $\boldsymbol{\lambda} \mathbf{1 0 9 1 4}$. We use $\log g f(\lambda 10327)=$ -0.35 and $\log g f(\lambda 10914)=-0.64$ according to Wiese \& Martin (1980). The recent results of Guet \& Johnson (1991) and Brage et al. (1998) give similar values: $\log g f$ $(\lambda 10327)=-0.30$ and -0.34 , respectively, and $\log g f$ $(\lambda 10914)=-0.59$ and -0.62 . The $C_{6}$-values for these lines (Table 3) have been computed with $\sigma_{0}$ and $\alpha$ taken from Barklem \& O'Mara (2000).

We compared different atomic models excluding and including $\mathrm{H}$ atom collisions with cross-sections calculated according to Steenbock \& Holweger (1984) and scaled by various factors $k_{\mathrm{H}}$. If hydrogen collisions are neglected we obtain for both lines broader and deeper theoretical profiles compared with the observed ones. Inclusion of these processes with $k_{\mathrm{H}}=0.1$ makes the NLTE profile shallower and narrower than the observed one. The best fits of both lines are obtained at $k_{\mathrm{H}}=0.01$. In Fig. 5 (bottom panel) we show one of these lines, $\lambda 10327$. For comparison the
Table 3. Atomic data for the Sr II lines. Most of the entries are self-explanatory; $f_{i j}$ of the HFS components correspond to $\mathrm{Sr}$ isotopic abundances of solar system matter.

\begin{tabular}{|c|c|c|c|c|}
\hline \multirow{2}{*}{$\begin{array}{c}\lambda[\AA] \\
\text { transition }\end{array}$} & \multicolumn{2}{|c|}{ HFS } & \multirow[t]{2}{*}{$\log \gamma_{R}$} & \multirow[t]{2}{*}{$\log C_{6}$} \\
\hline & $\Delta \lambda[\mathrm{m} \AA]$ & $f_{i j}$ & & \\
\hline 4215.534 & -17 & 0.0078 & 8.125 & -32.02 \\
\hline $5 \mathrm{~s}^{2} \mathrm{~S}_{1 / 2}-5 \mathrm{p}^{2} \mathrm{P}_{1 / 2}^{\circ}$ & -11 & 0.0052 & & \\
\hline \multirow[t]{4}{*}{ Variable syntax } & -1 & 0.0333 & & \\
\hline & 0 & 0.279 & & \\
\hline & 13 & 0.0028 & & \\
\hline & 18 & 0.0078 & & \\
\hline 4161.794 & & 0.19 & 8.529 & -31.40 \\
\hline \multicolumn{5}{|l|}{$5 \mathrm{p}^{2} \mathrm{P}_{1 / 2}^{\circ}-6 \mathrm{~s}^{2} \mathrm{~S}_{1 / 2}$} \\
\hline 10327.311 & & 0.074 & 8.175 & -31.60 \\
\hline \multicolumn{5}{|l|}{$4 \mathrm{~d}^{2} \mathrm{D}_{5 / 2}-5 \mathrm{p}^{2} \mathrm{P}_{3 / 2}^{\circ}$} \\
\hline 10914.877 & & 0.058 & 8.125 & -31.60 \\
\hline $4 \mathrm{~d}^{2} \mathrm{D}_{3 / 2}-5 \mathrm{p}^{2} \mathrm{P}_{1 / 2}^{\circ}$ & & & & \\
\hline
\end{tabular}

LTE profile corresponding to the same fitting parameters is presented, too. It is obvious that assuming LTE we cannot fit the $\lambda 10327$ line profile with reasonable values of $\log \varepsilon_{\mathrm{Sr}}$ and $V_{\text {mic }}$; even the line wings are affected by NLTE effects.

त4215. The Sr II resonance lines are affected by hyperfine structure (HFS). Strontium is represented by four stable isotopes. For solar system matter the ratio of the even $\mathrm{Sr}$ isotopes to the odd ones $\left({ }^{84} \mathrm{Sr}+{ }^{86} \mathrm{Sr}+{ }^{88} \mathrm{Sr}\right):{ }^{87} \mathrm{Sr}$ is 93:7 according to Cameron (1982). Isotopic shifts are very small $(\leq 2 \mathrm{~m} \AA)$ but the odd isotopes have hyperfine splitting of their levels resulting in several HFS components for a spectral line. We use the data on wavelengths and relative intensities of HFS components given by McWilliam et al. (1995). Oscillator strengths of separate components (Table 3 ) have been calculated using solar Sr isotopic abundances and $\log g f(\lambda 4215)=-0.172$ from Wiese \& Martin (1980). The most recent value $\log g f(\lambda 4215)=-0.175$ of Brage et al. (1998) coincides with that adopted in our study.

The Sr II $\lambda 4215.539 \AA$ line is blended by the strong Fe I $\lambda 4215.426 \AA$ line and by a few CN molecular lines in the far blue and red line wings. We treat Fe I $\lambda 4215$ with the fixed values of $\log g f=-1.76$ and $\log C_{6}=-30.69$. The last value was calculated using the above formula. For the Sun $\log \varepsilon_{\mathrm{Fe}}=7.51$ was adopted. Oscillator strengths of the CN molecular lines were fitted to reproduce the observed blend profile.

Sr II $\lambda 4215$ is strongly affected by van der Waals damping. The classical Unsöld (1955) formula gives $\log C_{6}=$ -32.275 while the formula above leads to $\log C_{6}=$ -31.805 with $\sigma_{0}$ and $\alpha$ from Barklem \& O'Mara (2000). Varying $\log C_{6}$ by only 0.1 has a significant effect on the total energy absorbed in this line. A careful analysis of 

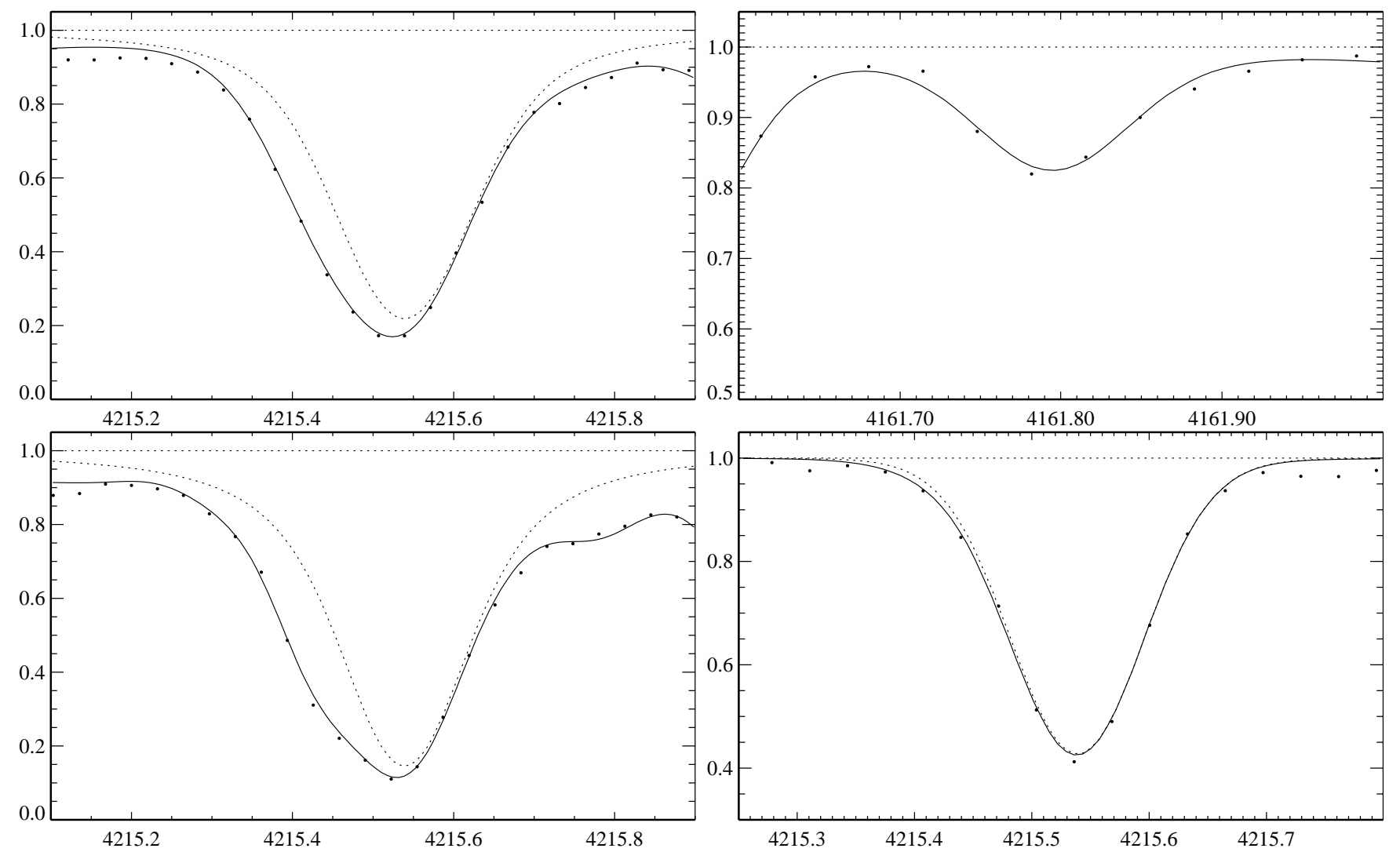

Fig. 6. NLTE synthetic (continuous line) and pure Sr II line profiles (dotted) compared with observed FOCES spectra (bold dots $)$ of HD $69611([\mathrm{Fe} / \mathrm{H}]=-0.60$, top row $), \mathrm{HD} 144579([\mathrm{Fe} / \mathrm{H}]=-0.69$, bottom row, left panel $)$ and HD $84937([\mathrm{Fe} / \mathrm{H}]=$ -2.07 , bottom row, right panel).

the solar line profile makes possible a separation of collisional broadening and blending effects. The best fit obtained with $\log C_{6}=-32.02$ is presented in Fig. 5 (top panel). For comparison we give also the pure Sr II $\lambda 4215$ NLTE profile calculated with the same parameters.

We did not succeed fitting the $\lambda 4215$ line core (Fig. 5) because it is formed in the uppermost atmospheric layers above $\log \tau_{5000}=-4$, and it is most probably influenced by a non-thermal and depth-dependent chromospheric velocity field that is not part of our solar model.

$\boldsymbol{\lambda 4 1 6 1}$. This line is located in the far red wing of two strong blends, Fe I $\lambda 4161.488 \AA$ and Ti II $\lambda 4161.534 \AA$. In addition, absorption in a few $\mathrm{CN}$ and $\mathrm{SiH}$ molecular lines near $4161.8 \AA$ lowers the continuum flux by about $5 \%$. We have found that $\log g f=-0.50$ given by Wiese \& Martin (1980) does not allow to reproduce the solar Sr II $\lambda 4161$ line with a fixed value of $\log \varepsilon_{\mathrm{Sr}}=2.92$ and reasonable values of $V_{\text {mic }}$. The best fit (Fig. 5, middle panel) is obtained with $\log g f=-0.41$ and $\log C_{6}=-31.4$. The last value is larger by 0.1 compared with the classical Unsöld (1955) constant.

\subsection{Stellar Sr abundances}

As mentioned above both Sr II lines of interest are blended. To obtain a good line profile fitting of the stellar spectra and, thus, to reduce Sr abundances errors we use only the spectra observed at $R \simeq 60000$ in 1998 to 2000 .
An exception refers to the four stars, HD 45282, HD 194598, HD 201891 and BD $66^{\circ} 268$, particularly important for our study. In total, Sr abundances have been determined for 49 stars and for 36 of them from both Sr II lines. The weaker $\lambda 4161$ line disappears at $[\mathrm{Fe} / \mathrm{H}]<-1$. As an example, we give in Fig. 6 the Sr II $\lambda 4215$ line profiles for the three metal-poor stars and the Sr II $\lambda 4161$ line profile for one of them. The contribution of the Fe I $\lambda 4215.426 \AA$ line blend reduces rapidly with decreasing $[\mathrm{Fe} / \mathrm{H}]$ because the electron number density affects line strengths of minor species such as Fe I much more than those of dominant ionization stages such as Sr II. It can be seen in Fig. 6 (right column, bottom panel) that for HD 84937 the contribution of the Fe I $\lambda 4215$ line is negligible. This holds also for the other 3 stars of our sample with $[\mathrm{Fe} / \mathrm{H}]<-1.9$ and $T_{\text {eff }}>6000 \mathrm{~K}$.

NLTE effects for the SrII lines are small for all the stars of our sample: NLTE abundance corrections $\Delta_{\text {NLTE }}$ are negative for $\lambda 4215$ and positive for $\lambda 4161$ and do not exceed 0.07 dex and 0.05 dex, respectively, by absolute value. For 36 stars with both Sr II lines investigated a difference of NLTE abundances derived from $\lambda 4215$ and $\lambda 4161$ is mainly within 0.08 dex with the mean value of $0.00 \pm 0.06$ dex while the mean difference of LTE abundances is $0.05 \pm 0.06$ dex.

In general, $\mathrm{Sr}$ abundances derived from the resonance line depend on the even-to-odd $\mathrm{Sr}$ isotope ratio adopted in calculations. We concluded in Paper I that $\mathrm{Ba}$ and $\mathrm{Eu}$ 


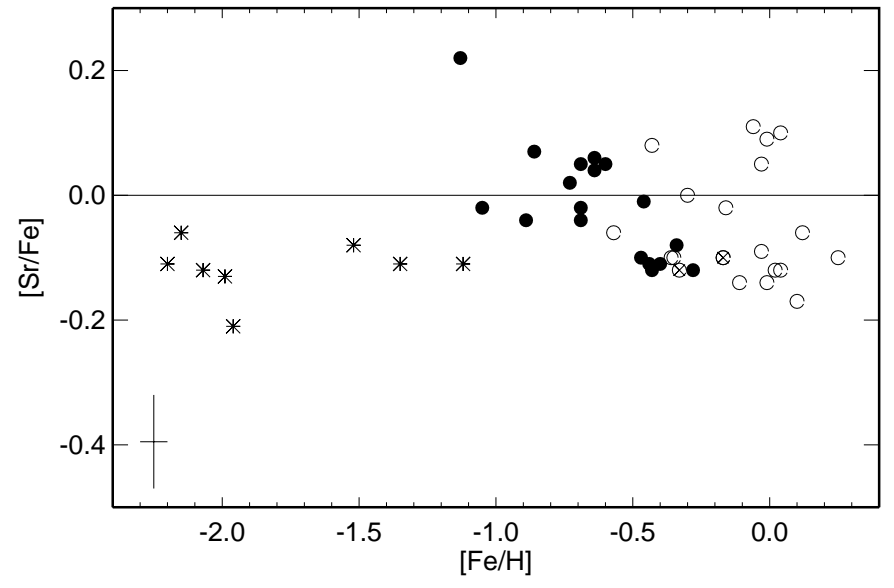

Fig. 7. The run of $[\mathrm{Sr} / \mathrm{Fe}]$ with $[\mathrm{Fe} / \mathrm{H}]$. Symbols are the same as in Fig. 1.

in halo and thick disk stars were mainly produced by $r$ process in high-mass stars. Sr might be produced not only in the $r$-process but also in a weak $s$-process that is related to high mass stars, too. According to Arlandini et al. (1999) the $r$-process contributes only to the ${ }^{88} \mathrm{Sr}$ isotope; the consequence of a dominating $r$-process is therefore the disappearance of HFS components in the Sr II lines. On the other hand the separation of HFS components of the $\lambda 4215$ line is not large ( $35 \mathrm{~m} \AA$ at maximum, see Table 3 ); Sr abundances derived from $\lambda 4215$ with and without HFS thus differ by 0.07 dex at $[\mathrm{Fe} / \mathrm{H}] \simeq-2$. According to Beer et al. (1992) the weak $s$-process produces much more even Sr isotopes than odd ones: $\left({ }^{86} \mathrm{Sr}+{ }^{88} \mathrm{Sr}\right):{ }^{87} \mathrm{Sr}=93: 7$, similarly to the main $s$-process which defines the solar system even-to-odd $\mathrm{Sr}$ isotope ratio. The ratio of the weak $s$ - to $r$-process is estimated as 3:2 (Arlandini et al. 1999). Consequently, the use of a solar even-to-odd $\mathrm{Sr}$ isotope ratio leads to an uncertainty of $\mathrm{Sr}$ abundances in halo and thick disk stars of not more than 0.02-0.03 dex. We neglect such a small value and use the solar even-to-odd $\mathrm{Sr}$ isotope ratio for all stars of our sample.

The final $[\mathrm{Sr} / \mathrm{Fe}]$ are presented in Table 1 and Fig. 7. Whenever both Sr II lines were available the average value was calculated. It can be concluded from Fig. 7 that the general behaviour of the $[\mathrm{Sr} / \mathrm{Fe}]$ abundance ratios with respect to metallicity is similar to that of $[\mathrm{Ba} / \mathrm{Fe}]$ (Fig. 1, top panel). For the thin disk stars there is a spread in $[\mathrm{Sr} / \mathrm{Fe}]$ up to 0.3 . Similarly to $[\mathrm{Ba} / \mathrm{Fe}]$ this points to a correlation of $[\mathrm{Sr} / \mathrm{Fe}]$ with stellar age: for 12 stars older than $5 \mathrm{Gyr}$ the mean value $\overline{[\mathrm{Sr} / \mathrm{Fe}]}=-0.10$ while for 7 stars younger than $5 \mathrm{Gyr} \overline{[\mathrm{Sr} / \mathrm{Fe}]}=0.04$. The thick disk stars show a decline of $[\mathrm{Sr} / \mathrm{Fe}]$ with $[\mathrm{Fe} / \mathrm{H}]$ increasing, so, that in "late" thick disk stars $([\mathrm{Fe} / \mathrm{H}]>-0.5)$ strontium is underabundant relative to iron by $0.1 \mathrm{dex}$, and this value coincides with the $\mathrm{Ba}$ underabundance reported in Sect. 3 for the thick disk stars. Underabundances of $\mathrm{Sr}$ relative to iron are typical for the halo stars which are close together with a mean value of $[\mathrm{Sr} / \mathrm{Fe}]=-0.10 \pm 0.02$. The discrepant result for $\mathrm{BD} 34^{\circ} 2476$ was dropped (see Sect. 6 for further discussion of this star).
The thick disk star, BD $0^{\circ} 2245([\mathrm{Fe} / \mathrm{H}]=-1.13)$, reveals a $\mathrm{Sr}$ overabundance relative to iron similar to that found for Ba (Sect. 3). There is no Hipparcos parallax for this star and the uncertainty of stellar parameters could explain apparent peculiar abundances of Sr and Ba. Another explanation would be that this star was the secondary component of a binary, and that we observe accreted $s$-process products formed in the evolved primary component.

There are only a few Sr abundance studies of cool stars in the literature. For the sample of 16 stars including dwarfs, giants and supergiants Gratton \& Sneden (1994) have found small Sr excess in the metallicity range from -0.9 down to -2.8 with the mean value $[\mathrm{Sr} / \mathrm{Fe}]=$ $0.07 \pm 0.11$ (10 stars) and slight underabundance of Sr relative to $\mathrm{Fe}$ up to 0.15 dex for 6 stars with $[\mathrm{Fe} / \mathrm{H}]>-0.6$. These data are based on the examination of equivalent widths of the Sr II $\lambda 4161$ line, and the authors note that the $[\mathrm{Sr} / \mathrm{Fe}]$ ratios given by the $\mathrm{Sr}$ II resonance line at $4215 \AA$ are smaller by $0.21 \pm 0.04 \mathrm{dex}$. The mean ratio $[\mathrm{Sr} / \mathrm{Fe}]=-0.14$ deduced from $\lambda 4215$ line for the halo stars is in agreement with that found in the present study. At $[\mathrm{Fe} / \mathrm{H}]<-1$ the Sr II subordinate line is rather weak, and in our opinion $\mathrm{Sr}$ abundances based on the Sr II $\lambda 4215$ line are more reliable than those derived from Sr II $\lambda 4161$. Using equivalent widths of Sr II $\lambda 4077$ and $\lambda 4215$ for a sample of cool dwarfs in the metallicity range similar to ours, Hartmann \& Gehren (1988) have obtained [Sr/Fe] abundance ratios close to solar, independent of the general metal abundance. However, the large scatter of up to 0.5 dex masks any features in the run $[\mathrm{Sr} / \mathrm{Fe}]$ vs. $[\mathrm{Fe} / \mathrm{H}]$. Based on the Sr I $\lambda 4607$ line Jehin et al. (1999) have obtained $[\mathrm{Sr} / \mathrm{Fe}]$ abundance ratios between 0 and -0.4 for a sample of 21 mildly metal-poor stars in the narrow metallicity range from -0.8 down to -1.3 . We note that using the LTE assumption may result in an underestimate of element abundances derived from spectral lines of minor species such as Sr I. Magain (1989) has studied only metal-poor stars with $[\mathrm{Fe} / \mathrm{H}]<-1.4$ and obtained a Sr excess of about 0.4 dex at $[\mathrm{Fe} / \mathrm{H}]$ between -1.5 and -2.5 and a decline of the $[\mathrm{Sr} / \mathrm{Fe}]$ abundance ratios at lower metallicities, however, he notes that results for Sr should be considered as preliminary due to the strength of the available lines (Sr II $\lambda 4077$ and $\lambda 4215$ ) and the uncertainties affecting the $g f$-values as well as the damping constants. In the range of overlapping metallicities his data are different from ours. For extremely metal-poor stars with $[\mathrm{Fe} / \mathrm{H}]<-2.4$ McWilliam et al. (1995) and Ryan et al. (1996) have found a decline of the [Sr/Fe] abundance ratios with decreasing metallicity and a spread in these ratios up to 2.5 dex. Elemental abundances were determined from the Sr II $\lambda 4077$ and $\lambda 4215$ lines with the LTE assumption. It was noted in Sect. 4 that for extremely metal-poor stars NLTE effects for the Sr II lines depend strongly on stellar parameters. We give one example. For two stars of Ryan et al. sample, BS 16968-061 $\left(T_{\text {eff }}=6000 \mathrm{~K}, \log g=\right.$ $4,[\mathrm{Fe} / \mathrm{H}]=-3.08)$ and CS 22186-005 $\left(T_{\text {eff }}=6000 \mathrm{~K}\right.$, $\log g=2,[\mathrm{Fe} / \mathrm{H}]=-2.77)$, we have computed NLTE 

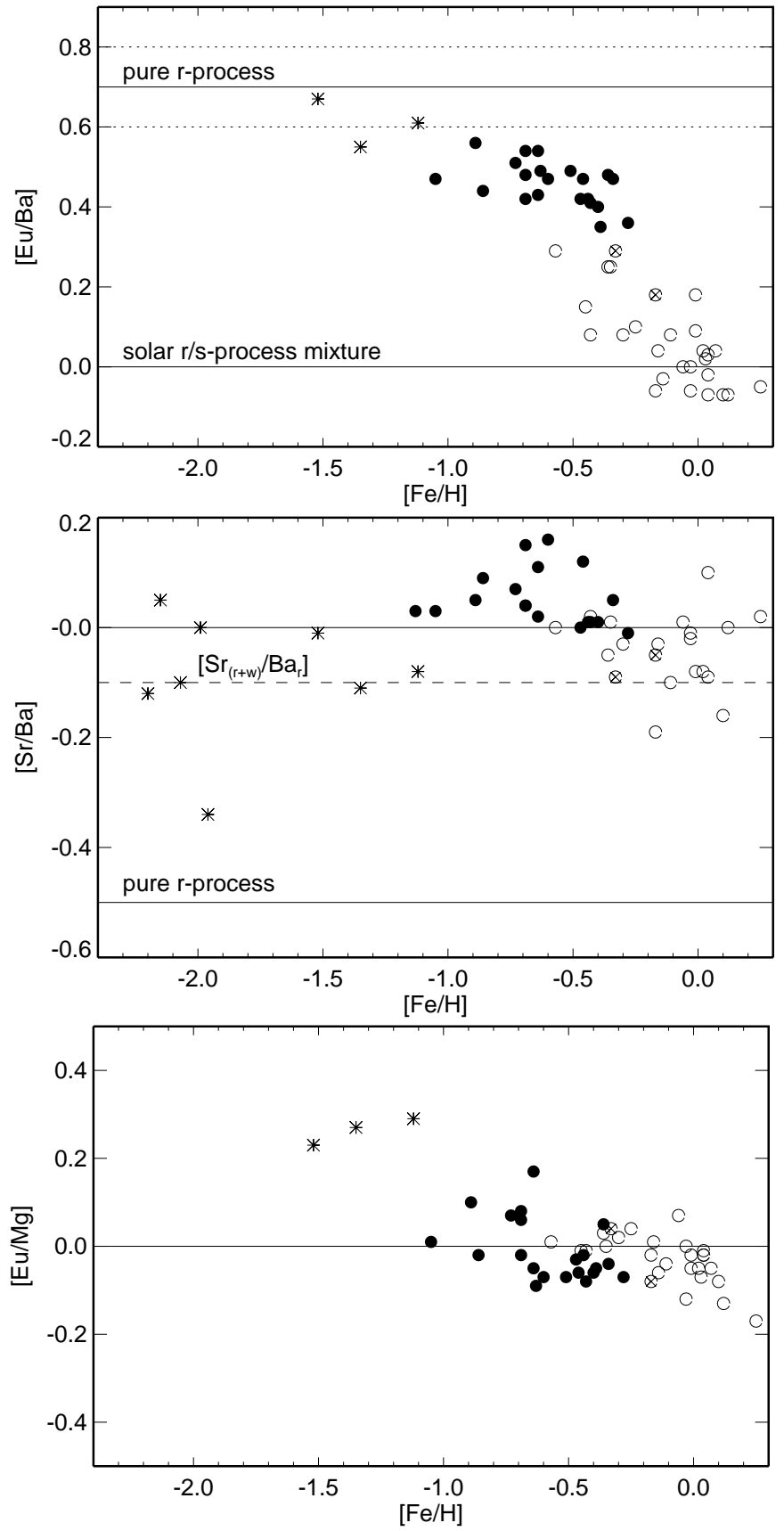

Fig. 8. Variation of element abundance ratios with $[\mathrm{Fe} / \mathrm{H}]$. Symbols are the same as in Fig. 1. Dotted lines in the top panel limit the range of the $[\mathrm{Eu} / \mathrm{Ba}]_{r}$ ratio uncertainty.

abundance corrections $\Delta_{\mathrm{NLTE}}=0.25$ dex and $0.60 \mathrm{dex}$, respectively. Based on NLTE Sr abundances we obtain for these stars new values $[\mathrm{Sr} / \mathrm{Fe}]=-0.25$ and -0.63 instead of -0.50 and -1.23 as determined by Ryan et al. Therefore, the large spread in $[\mathrm{Sr} / \mathrm{Fe}]$ ratios published by McWilliam et al. (1995) and Ryan et al. (1996) may be at least in part due to neglecting NLTE effects for the Sr II lines.

\section{Element abundance ratios [Eu/Ba], [Eu/Mg], [Sr/Ba], and the evolution of the Galaxy}

In this section we discuss the $[\mathrm{Eu} / \mathrm{Ba}],[\mathrm{Eu} / \mathrm{Mg}]$ and $[\mathrm{Sr} / \mathrm{Ba}]$ abundance ratios (Fig. 8) in the halo, thick and thin disk of the Galaxy. These ratios provide useful diagnostics of the role of different processes ( $\alpha$-process, weak and main $s$-process, $r$-process) in the chemical enrichment of the interstellar gas and thus give important information about dominant sites of nucleosynthesis at different epochs of Galactic evolution.

There is strong evidence that the relative elemental (at least, with $Z<70) r$-process abundances have not changed over the history of the Galaxy. Sneden et al. (1996) have found the elemental abundances in the extremely metal-poor star CS 22892-052 $([\mathrm{Fe} / \mathrm{H}] \simeq-3.1)$ consistent with the solar $r$-process distribution for the elements $Z \geq 56$. Similar results have been obtained for another three halo stars $([\mathrm{Fe} / \mathrm{H}]=-2.7$ and -1.7$)$ by Cowan et al. (1999) and for the three stars in the globular cluster M $15([\mathrm{Fe} / \mathrm{H}]=-2.2)$ by Sneden et al. (2000). Hill et al. (2001) studied element abundances in the range $Z=38$ to $Z=92$ in the halo star CS 31082-001 with $[\mathrm{Fe} / \mathrm{H}]=-2.9$ and concluded that the $56<Z<70$ elements are very well reproduced by a solar $r$-process. In Fig. 8 (top panel) we include a line indicating the solar abundance ratio of $\mathrm{Eu}$ to $\mathrm{Ba}$ contributed by the $r$ process (Arlandini et al. 1999) relative to the total abundances, $[\mathrm{Eu} / \mathrm{Ba}]_{r}=0.70$. This value is uncertain within 0.1 dex mainly due to an uncertainty of the $r$-process contribution to ${ }^{138} \mathrm{Ba}$ estimated by Arlandini et al. (1999) as $58 \%$. To obtain the pure $r$-process $[\mathrm{Sr} / \mathrm{Ba}]_{r}$ abundance ratio the contributions of the main and weak $s$-process to solar Sr have to be evaluated. The main $s$-process contributes $85 \%$ according to Arlandini et al. (1999). Up to now there is no realistic model of the weak $s$-process and Arlandini et al. estimate the ratio between the weak $s$ process and $r$-process contributions to solar $\mathrm{Sr}$ as 3:2 using the schematic approach of Beer et al. (1992). This gives $[\mathrm{Sr} / \mathrm{Ba}]_{r}=-0.50$, while the solar ratio of $\mathrm{Sr}$ contributed altogether by the $r$ - and weak $s$-process to Ba contributed by the $r$-process, $\left[\mathrm{Sr}_{w+r} / \mathrm{Ba}_{r}\right]$, equals -0.10 . The last value is determined with less uncertainty of about 0.1 dex compared with 0.2 dex or even more for the $[\mathrm{Sr} / \mathrm{Ba}]_{r}$ ratio.

Our results, apparent from Fig. 8, confirm in general and improve the conclusions drawn in Paper I; they also provide the fundaments of new conclusions.

Europium is overabundant relative to barium in halo stars with a mean value $[\mathrm{Eu} / \mathrm{Ba}]=0.61$. We have analyzed only three halo stars and this limits us in drawing reliable conclusions concerning the halo. However, these stars complement from the side of a moderate metal-deficiency the sample of 14 extremely metal-poor stars with $[\mathrm{Fe} / \mathrm{H}] \leq$ -2.4 studied by McWilliam (1998). Using the LTE assumption he has obtained a mean value $[\mathrm{Eu} / \mathrm{Ba}]=0.69$. At stellar parameters typical for his sample our NLTE calculations for Ba II and Eu II show positive NLTE abundance corrections for both elements but their values are 
larger for barium. So, the mean value $[\mathrm{Eu} / \mathrm{Ba}]=0.69$ found by McWilliam might be smaller by about $0.05-$ 0.1 dex, and our data for the moderately metal-deficient halo stars show the same $[\mathrm{Eu} / \mathrm{Ba}]$ ratio. Thus, the observed $[\mathrm{Eu} / \mathrm{Ba}]$ ratios, close to $[\mathrm{Eu} / \mathrm{Ba}]_{r}=0.70$ independent of metallicity, favour the dominance of the r-process heavy element synthesis during the formation of the halo population. If the upper mass limit of AGB stars' progenitors, responsible for $\mathrm{Ba}$ synthesis in the $s$-process, lies between $3 M_{\odot}$ (Raiteri et al. 1999) and $4 M_{\odot}$ (Travaglio et al. 1999) $s$-nuclei of Ba appear after about 0.3-0.6 Gyr from the beginning of the protogalactic collapse. From the insignificant contribution of the $s$-process to Ba production we conclude that the halo population has formed rapidly during an interval of 0.3-0.6 Gyr.

Strontium is slightly underabundant relative to barium in halo stars with a mean value of $[\mathrm{Sr} / \mathrm{Ba}]=-0.05 \pm$ 0.06 (except for BD $34^{\circ} 2476$ ). For most stars of his sample McWilliam (1998) has obtained positive or close to $0[\mathrm{Sr} / \mathrm{Ba}]$ abundance ratios. Thus, $[\mathrm{Sr} / \mathrm{Ba}]$ in halo stars is much higher compared with $[\mathrm{Sr} / \mathrm{Ba}]_{r}=-0.50$, and a secondary source of Sr must have occurred. Could the weak $s$-process be that source? Similarly to the $r$-process it runs in high-mass stars with an evolution time consistent with the short timescale for the halo. On the other hand, the amount of Sr produced by the weak $s$-process is expected to be very low in metal-poor stars simply due to the secondary nature of the weak $s$-process. We note that halo stars reveal $[\mathrm{Sr} / \mathrm{Ba}$ ] abundance ratios close to $\left[\mathrm{Sr}_{w+r} / \mathrm{Ba}_{r}\right]=-0.10$. Is this similarity accidental? Or is it because the efficiency of the weak $s$-process becomes more significant at $[\mathrm{Fe} / \mathrm{H}] \geq-2$ ? An alternative possibility would be that the second $\mathrm{Sr}$ source is the $\alpha$-rich freeze-out, as detailed by Woosley \& Hoffman (1992); this mechanism is predicted to synthesize elements up to the Sr region, and as a primary process should not be extinguished at low metallicity. More theoretical work will be required to answer these questions.

Only one halo star, BD $34^{\circ} 2476$, reveals a clear Sr underabundance relative to $\mathrm{Ba}$ with $[\mathrm{Sr} / \mathrm{Ba}]=-0.34$. The two CH subgiants from McWilliam's (1998) sample show $[\mathrm{Sr} / \mathrm{Ba}]<-0.4$. Does it mean these stars formed far from the weak $s$-process sites?

Europium is overabundant relative to barium in thick disk stars with $[\mathrm{Eu} / \mathrm{Ba}]$ abundance ratios between 0.56 and 0.35 . We first note a slight decline of this ratio with increasing metallicity: $[\mathrm{Eu} / \mathrm{Ba}]$ reduces by about $0.1-$ 0.15 dex as $[\mathrm{Fe} / \mathrm{H}]$ grows from -1 to -0.3 . This means that the r-process remained dominant in heavy element production during thick disk evolution. At the same time, evolved low mass stars started to enrich the interstellar gas by $s$-nuclei of $B a$. The decrease of the $[\mathrm{Eu} / \mathrm{Ba}]$ ratio by $0.1-0.15$ dex constitutes a constraint to the duration of that phase. From the small contribution of the $s$ process to $\mathrm{Ba}$ production we conclude that, similar to the halo, the thick disk population formed in the early Galaxy, when high mass stars were the main sites of nucleosynthesis. Keeping in mind the evolution time of AGB star progenitors we suppose that the duration of halo and thick disk formation was not much longer than 1 Gyr. This conclusion is in good agreement with ages of the three thick disk subgiants of our sample obtained by Bernkopf et al. (2001) on the base of recently improved stellar interior calculations. Bernkopf et al. (2001) give $13.8 \pm 1.3 \mathrm{Gyr}$, $13.5 \pm 1.3$ Gyr and $12.5 \pm 1.1$ Gyr for the thick disk subgiants HD 3795, HD 10519 and HD 222794, respectively. It is also compatible with age estimates for halo stars based on the detection of the Th II $\lambda 4019$ line in spectra of very metal-poor stars of Sneden et al. (1996) and Cowan et al. (1999) who have obtained an average age of $15.6 \pm$ 4.6 Gyr for the two stars CS 22892-052 $([\mathrm{Fe} / \mathrm{H}] \simeq-3.1)$ and $\operatorname{HD} 115444([\mathrm{Fe} / \mathrm{H}] \simeq-2.7)$; Sneden et al. (2000) also have determined an age of $14 \pm 3$ Gyr for the stars in M 15. Recently Cayrel et al. (2001) have detected the U II $\lambda 3859.57$ line in the very metal-poor star CS 31082-0018, and using uranium abundance as a cosmochronometer have estimated an age of this star as $12.5 \pm 3$ Gyr. As Spite (2001) kindly informed us, the $g f$-value of the U II line has been revised since the publication of Cayrel et al. (2001) result and the age is now $13.2 \pm 2$ Gyr. Thus, within error bars the thick disk stellar population is as old as the halo. We note further that the halo and thick disk stars' ages agree well with the recent cosmological age estimates, based on high-redshift supernovae, of 14.9 \pm 1.5 Gyr (Perlmutter et al. 1999) and $14.2 \pm 1.7$ Gyr (Riess et al. 1998).

Strontium is slightly overabundant relative to barium in the thick disk stars with the mean value $[\mathrm{Sr} / \mathrm{Ba}]=0.05 \pm 0.05$. This could be due to a strengthening of the weak $s$-process with increasing overall metallicity. Theoretical studies of the weak $s$-process are required to test this idea.

This study confirms the step-like decrease of the $[E u / B a]$ abundance ratio at the thick-to-thin disk transition found in our previous analysis (Paper I). In the region of overlapping metallicities the $[\mathrm{Eu} / \mathrm{Ba}]$ ratios in the thin disk stars are lower on average by 0.25 dex compared with the thick disk stars. This finding is indicative of a phase of ceased star formation before the onset of the thin disk formation during which $r$-process element production stopped but $s$-process nuclei of Ba were synthesized in evolved low mass stars. The duration of this intermediate phase can be evaluated from calculations of the s-process nucleosynthesis in $A G B$ stars, and our $[\mathrm{Ba} / \mathrm{Fe}]$ (Fig. 1) and [Eu/Ba] (Fig. 8) ratios provide observational constraints. Such a hiatus in star formation was suggested by Gratton et al. (1996) and Fuhrmann (1998) on the base of the $[\alpha / \mathrm{Fe}]$ abundance ratio analyses. Direct evidence of a star formation gap between thick and thin disk of no less than 3 Gyr is given by Bernkopf et al. (2001). They have obtained stellar ages between 6.8 and 8.1 Gyr for the three thin disk subgiants and between 12.5 and $13.8 \mathrm{Gyr}$ for the thick disk subgiants mentioned above. Thus, from the point of view of their chemical history and age the thick disk stellar population is much closer to the halo than to the thin disk stellar population. 
The thin disk stars show a steep decline of the $[\mathrm{Eu} / \mathrm{Ba}]$ abundance ratios with increasing metallicity: $[\mathrm{Eu} / \mathrm{Ba}]$ is reduced by about 0.35 dex as one goes from $[\mathrm{Fe} / \mathrm{H}]=-0.5$ to 0.25 . These data indicate that evolved low mass stars now produce larger masses of $s$-process elements as compared with the return of $r$-elements from high mass stars, well in agreement with the thin disk IMF and the long timescale of about 9 Gyr. Sr nearly follows Ba in the thin disk stars and this confirms again that the main $s$-process now becomes dominant in the production of these elements.

In Paper I we have first reported an overabundance of $\mathrm{Eu}$ relative to $\mathrm{Mg}$ in two halo stars. One halo star added in this study, HD 103095, shows the same overabundance with $[\mathrm{Eu} / \mathrm{Mg}]=0.27$. Moreover, there is a marginal tendency towards a higher $[\mathrm{Eu} / \mathrm{Mg}]$ ratio in the "early" thick disk stars (the mean value $[\mathrm{Eu} / \mathrm{Mg}]=0.04 \pm 0.05$ at $[\mathrm{Fe} / \mathrm{H}]<-0.65)$ compared with the "late" thick disk stars (the mean value $[\mathrm{Eu} / \mathrm{Mg}]=-0.05 \pm 0.04$ at the near solar Mg abundances, except for HD 3795 with $[\mathrm{Eu} / \mathrm{Mg}]=$ $0.17)$.

The knowledge of the $[\mathrm{Eu} / \mathrm{Mg}]$ abundance ratio in the oldest stars of the Galaxy is of great importance for an estimate of the timescale for early Galaxy formation. Theoretical predictions of SN II element yields show that $[\alpha / \mathrm{Fe}]$ increases with increasing progenitor mass (Arnett 1991). Most theoretical models of $r$-process nucleosynthesis are based on low mass $\left(8-12 M_{\odot}\right)$ supernovae (Mathews \& Cowan 1990; Tsujimoto \& Shigeyama 1998; Travaglio et al. 1999). Ishimaru \& Wanajo (1999) constrain the mass range of SNe for the $r$-process site by either 8-10 $M_{\odot}$ or $\geq 30 M_{\odot}$. If the production of Eu is related to low mass $\mathrm{SNe}$ while $\mathrm{Mg}$ is produced in larger amounts in high-mass $\mathrm{SNe}$ we should expect an underabundance and, certainly, not an overabundance of Eu relative to $\mathrm{Mg}$ in the oldest stars of the Galaxy. We have inspected europium and magnesium abundances available in the literature. For a sample of 12 halo stars with $[\mathrm{Fe} / \mathrm{H}]$ from -2.66 to -1.48 from Magain's (1989) work the $[\mathrm{Eu} / \mathrm{Mg}]$ abundance ratios vary from -0.01 to 0.51 with the mean value $[\mathrm{Eu} / \mathrm{Mg}]=0.18$. Only one star, $\mathrm{HD} 140283$, shows an underabundance of Eu relative to $\mathrm{Mg}$ of $0.30 \mathrm{dex}$, however, for the same star Ryan et al. (1996) give $[\mathrm{Eu} / \mathrm{Mg}]=$ 0.33 . A surprisingly large spread in $[\mathrm{Eu} / \mathrm{Mg}]$ can be found in the McWilliam et al. (1995) and Ryan et al. (1996) data for very metal-poor stars. In the first paper the $[\mathrm{Eu} / \mathrm{Mg}]$ ratios vary from -0.52 to 1.95 for the sample of 14 stars and in the second one from 0.15 to 1.86 for a sample of 12 stars with one star revealing $[\mathrm{Eu} / \mathrm{Mg}]=$ -1 . At the same time, we note a large divergence of elemental abundances between these two studies for stars in common. For example, for CS 22952-015 ([Fe/H $\simeq-3.4)$ McWilliam et al. and Ryan et al. obtain $[\mathrm{Mg} / \mathrm{Fe}]=-0.18$ and 0.38 , respectively; for $\mathrm{CS} 22968-014([\mathrm{Fe} / \mathrm{H} \simeq-3.4)$ $[\mathrm{Mg} / \mathrm{Fe}]=-0.06$ and 0.64 ; for $\mathrm{CS} 22885-096([\mathrm{Fe} / \mathrm{H} \simeq$ -3.8) Ryan et al. find an overabundance of Eu relative to Fe of 1 dex while McWilliam et al. cannot even measure the Eu II lines. Thus, much more observational work will be required to improve europium to magnesium abundance ratios in halo stars. A large spread in $[\mathrm{Eu} / \mathrm{Mg}]$ for very metal-poor stars, if it exists, indicates different sites for $\mathrm{Mg}$ and Eu production and quite insufficient mixing of the interstellar gas in the early Galaxy.

As our sample of halo stars is small ( 3 stars) we can draw only a preliminary conclusion that our data on the $[\mathrm{Eu} / \mathrm{Mg}]$ abundance ratios in the halo and "early" thick disk stars complemented by the data available in the literature do not support theoretical models of the r-process based on low mass SNe. Assume that $\mathrm{Eu}$ is mostly produced in the higher mass $\mathrm{SNe}$ compared with $\mathrm{Mg}$. In this case a timescale for the galactic halo is defined by a time delay of SNe II producing $\mathrm{Mg}$ and it cannot be larger than 20 million years which is the evolution time of $8 M_{\odot}$ mass star (Massevich \& Tutukov 1988). Therefore the halo formation phase may indeed be much shorter than the 0.30.6 Gyr deduced above from the analysis of the $[\mathrm{Eu} / \mathrm{Ba}]$ abundance ratios.

Summing up the above results we imagine the following scenario of the Galaxy evolution. The first stellar population of the Galaxy consisted of very high-mass stars and produced heavy elements with a higher efficiency for the $r$-process elements compared with $\alpha$-elements or iron. Thus the interstellar gas, out of which the second stellar population (halo) formed, had $[\mathrm{Eu} / \mathrm{Mg}]>0,[\mathrm{Eu} / \mathrm{Fe}]>$ 0 and $[\mathrm{Mg} / \mathrm{Fe}]>0$. The halo stellar population formed during a very short interval comparable to the evolution time of progenitors of those SNe II responsible for $\mathrm{Mg}$ production. The question then is: what are masses of these progenitors? The halo formation was characterized by a very high star formation rate. During this phase $r-, \alpha$-elements and iron were produced with nearly the same efficiency and, probably, in common sites, so that the $[\mathrm{Mg} / \mathrm{Fe}],[\mathrm{Eu} / \mathrm{Fe}]$ and $[\mathrm{Eu} / \mathrm{Mg}]$ ratios kept their values. Until the onset of thick disk formation the progenitors of SNe II, which are the major producers of magnesium, have evolved and the $[\mathrm{Eu} / \mathrm{Mg}]$ abundance ratio decreases in the "early" thick disk stars (Fig. 8, bottom panel). As the evolution time of SNe II progenitors is not longer than 20 million years therefore the onset of the thick disk refers to the early Galaxy. The timescale for the thick disk formation is probably of the order of $1 \mathrm{Gyr}$. During this phase iron starts to be produced in SNe I and its production rate is higher than that for Eu resulting in a steep decline of $[\mathrm{Eu} / \mathrm{Fe}]$ with $[\mathrm{Fe} / \mathrm{H}]$ (Fig. 2, top panel); the production rate of iron is also higher than for $\mathrm{Mg}$ (there is evidence of a slight decline of the $[\mathrm{Mg} / \mathrm{Fe}]$ and $[\alpha / \mathrm{Fe}]$ abundance ratios with metallicity in the figures of Bernkopf et al. 2001; Prochaska et al. 2000). The heavy elements beyond the iron group are mainly produced by the $r$-process in high mass SNe II, however, the main $s$-process nuclei appear. The decrease of the $[\mathrm{Eu} / \mathrm{Ba}]$ ratio by about $0.1-0.15$ dex implies a constraint to the duration of the thick disk formation phase. Then star formation in our Galaxy stopped for about 3 Gyr (according to Bernkopf et al. 2001). Europium abundances $[\mathrm{Eu} / \mathrm{H}]$ (Fig. 2, bottom panel) and $\mathrm{Mg}$ abundances $[\mathrm{Mg} / \mathrm{H}]$ 
(Bernkopf et al. 2001) remained constant during this phase while iron and the main $s$-process elements such as Ba continued to be produced in evolved lower-mass stars. The decrease by about 0.25 dex of the $[\mathrm{Eu} / \mathrm{Ba}]$ ratio at the thickto-thin disk transition provides an independent method to estimate the duration of that intermediate phase. The thin disk phase then was characterized by the higher iron production rate compared with that for $\alpha$ - (Fuhrmann 1998) and $r$-elements (Fig. 2, top panel). In turn, the main $s$-process elements were produced during this phase with a larger efficiency compared with iron (Table 2).

The suggested scenario based on the chemical history of the Galaxy will be useful to develop a realistic model of the Galaxy evolution taking into consideration physical and dynamical parameters of the galactic stellar populations. One of the important and unsolved problems of the Galaxy's chemical evolution concerns the astrophysical site for the $r$-process; it requires further work in stellar evolution, nuclear physics and stellar spectroscopy. Yet it is somewhat surprising that 40 years after the trailblazing work of Eggen et al. (1962) we return to very much the same conclusions, however, with at least one more population than was known at that time.

Acknowledgements. M. L. acknowledges with gratitude the Max-Planck-Institut of Astrophysics for the partial support of this study and the Institute of Astronomy and Astrophysics of Munich University for warm hospitality during a productive stay in July-September 2000. M. L. thanks especially RolfPeter Kudritzki for his support. We thank Klaus Fuhrmann for providing reduced FOCES spectra and parameters for most of the stars investigated in this paper, for valuable help and useful discussions, and for comments on the manuscript of this paper. We are grateful to Andreas Korn for providing reduced FOCES spectra and parameters for the three stars and to Johannes Reetz for providing the SIU code for synthetic spectrum computations. We report with sorrow the death of Michael Pfeiffer who designed and built the échelle spectrograph FOCES, on which our present results are based. M. L. has been partially supported by the Russian Basic Researches Fund (grant 99-0217488).

\section{References}

Allen, C. W. 1973, Astrophysical Quantities (Athlone Press)

Anders, E., \& Grevesse, N. 1989, Geoch. Cosmochim. Acta, 53, 197

Anstee, S. D., \& O'Mara, B. J. 1995, MNRAS, 276, 859

Arlandini, C., Käppeler, F., Wisshak, K., et al. 1999, ApJ, 525, 886

Arnett, D. 1991, in Frontiers of Stellar Evolution, ASP Conf. Ser., 20, 389

Auer, L. H., \& Heasley, J. 1976, ApJ, 205, 165

Barklem, P. S., \& O'Mara, B. J. 1997, MNRAS, 290, 102

Barklem, P. S., O'Mara, B. J., \& Ross, J. E. 1998, MNRAS, 296, 1057

Barklem, P. S., \& O'Mara, B. J. 2000, MNRAS, 311, 535
Beer, H., Walter, G., \& Käppeler, F. 1992, ApJ, 389, 784

Belyakova, E. V., \& Mashonkina, L. I. 1997, Astron. Rep., 41, 530

Bernkopf, J., Fiedler, A., \& Fuhrmann, K. 2001, in Astrophysical Ages and Time Scales, ASP Conf. Ser., in press

Brage, T., Wahlgren, G. M., Leckrone, D. S., \& Proffitt, C. R. 1998, ApJ, 496, 1051

Cameron, A. G. W. 1982, Ap\&SS, 82, 123

Cayrel, R., Hill, V., Beers, T. C., et al. 2001, Nature, 409, 691

Chen, Y. Q., Nissen, P. E., Zhao, G., Zhang, H. W., \& Benoni, T. 2000, A\&AS, 141, 491

Cowan, J. J., Pfeiffer, B., Kratz, K.-L., et al. 1999, ApJ, 521, 194

Drawin, H.-W. 1961, Z. Phys., 164, 513

Drawin, H.-W. 1968, Z. Phys., 211, 404

Drawin, H.-W. 1969, Z. Phys., 225, 483

Edvardsson, B., Andersen, J., Gustafsson, B., et al. 1993, A\&A, 275,101

Eggen, O. J., Linden-Bell, D., \& Sandage, A. 1962, ApJ, 136, 748

Fuhrmann, K. 1998, A\&A, 338, 161

Fuhrmann, K. 2001, in preparation

Gilmore, G., \& Reid, N. 1983, MNRAS, 202, 1025

Gratton, R. G., \& Sneden, C. 1994, A\&A, 287, 927

Gratton, R. G., Carretta, E., Matteucci, F., \& Sneden, C. 1996, ASP Conf. Ser., 92, 307

Gratton, R. G., Carretta, E., Matteucci, F., \& Sneden, C. 2000, A\&A, 358, 671

Guet, C., \& Johnson, W. R. 1991, Phys. Rev., A44, 1531

Grevesse, N., Noels, A., \& Sauval, A. J. 1996, ASP Conf. Ser., 99, 117

Hartmann, K., \& Gehren, T. 1988, A\&A, 199, 269

Hill, V., Plez, B., Cayrel, R., \& Beers, T. C. 2001, in Astrophysical Ages and Time Scales, ASP Conf. Ser., in press

Holweger, H. 1979, in Les Éléments et leurs Isotopes dans l'Univers, 22nd Liège Symp., Liège, 117

Ishimaru, Y., \& Wanajo, S. 1999, ApJ, 511, L33

Jehin, E., Magain, P., Neuforge, C., et al. 1999, A\&A, 341, 241

Käppeler, F., Beer, H., \& Wisshak, K. 1989, Rep. Prog. Phys., 52,945

Korn, A. 2000, private communication

Kurucz, R. L. 1994, CD-ROM, 18, 19

Kurucz, R. L., Furenlid, I., Brault J., \& Testerman L. 1984, Solar Flux Atlas from 296 to 1300 nm, Nat. Solar Obs., Sunspot (New Mexico)

Lindgard, A., \& Nielsen, S. E. 1977, Atomic Data Nucl. Data Tables, 19, 533

Magain, P. 1989, A\&A, 209, 211

Mashonkina, L. I. 2000, Astron. Rep., 44, 558

Mashonkina, L. I., \& Bikmaev, I. F. 1996, Astron. Rep., 40, 109

Mashonkina, L. I., Gehren, T., \& Bikmaev, I. F. 1999, A\&A, 343,519

Mashonkina, L. I., \& Gehren, T. 2000, A\&A, 364, 249, Paper I

Massevitch, A. G., \& Tutukov, A. V. 1988, Stellar Evolution: theory and observations (Moscow: "Nauka")

Mathews, G. J., \& Cowan, J. J. 1990, Nature, 345, 491

McWilliam, A. 1998, AJ, 115, 1640

McWilliam, A., Preston, G. W., Sneden, C., \& Searle, L. 1995, AJ, 109, 2757

Moore, C. E. 1952, Atomic Energy Levels. Circ. NBS, 467 
Peach, G. 1967, Mem. R. Astron. Soc., 71, 13

Perlmutter, S., Aldering, G., Goldhaber, G., et al. 1999, ApJ, 517,565

Pfeiffer, M., Frank, C., Baumüller, D., Fuhrmann, K., \& Gehren, T. 1998, A\&AS, 130, 381

Prochaska, J. X., Naumov, S. O., Carney, B. W., McWilliam, A., \& Wolfe, A. M. 2000, AJ, 120, 2513

Raiteri, C. M., Villata, M., Gallino, R., Busso, M., \& Cravanzola, A. 1999, ApJ, 518, L91

Riess, A. G., Filippenko, A. V., Challis, P., et al. 1998, AJ, 116, 1009

Ryan, S. G., Norris, J. E., \& Beers, T. C. 1996, ApJ, 471, 254

Sakhibullin, N. A. 1983, Trudi Kazan Gor. Obs., 48, 9

Sneden, C., McWilliam, A., Preston, G. W., et al. 1996, ApJ, 467,819
Sneden, C., Johnson, J., Kraft, R. P., et al. 2000, ApJ, 536, L85

Spite, M. 2001, private communication

Steenbock, W., \& Holweger, H. 1984, A\&A, 130, 319

Travaglio, C., Galli, D., Gallino, R., et al. 1999, ApJ, 521, 691

Tsujimoto, T., \& Shigeyama, T. 1998, ApJ, 508, L151

Unsöld, A. 1955, Physik der Sternatmosphären, 2nd edition (Springer, Berlin - Göttingen - Heidelberg)

VandenBerg, D. A., Swenson, F. J., Rogers, F. J., Iglesias, C. A., \& Alexander, D. R. 2000, ApJ, 532, 430

van Regemorter, H. 1962, ApJ, 136, 906

Wiese, W. L., \& Martin, G. A. 1980, Wavelengths and Transition Probabilities for Atoms and Atomic Ions. Part II. NSRDS - NBS 68 (Washington, DC)

Woosley, S. E., \& Hoffman, R. D. 1992, ApJ, 395, 202 